\title{
Orion MPCV Continuum RCS Heating Augmentation Model Development
}

\author{
Andrew J. Hyatt* \\ ERC, Inc., NASA Ames Research Center, Moffett Field, CA 94035 \\ Molly E. White ${ }^{\dagger}$ \\ NASA Johnson Space Center, Houston, TX 77058
}

\begin{abstract}
The reaction control system jets of the Orion Multi Purpose Crew Vehicle can have a significant impact on the magnitude and distribution of the surface heat flux on the leeside of the aft-body, when they are fired. Changes in surface heating are expressed in terms of augmentation factor over the baseline smooth body heating. Wind tunnel tests revealed heating augmentation factors as high as 13.0, 7.6, 2.8, and 5.8 for the roll, pitch down, pitch up, and yaw jets respectively. Heating augmentation factor models, based almost exclusively on data from a series of wind tunnel tests have been developed, for the purposes of thermal protection system design. The wind tunnel tests investigated several potential jet-to-freestream similarity parameters, and heating augmentation factors derived from the data showed correlation with the jet-to-freestream momentum ratio. However, this correlation was not utilized in the developed models. Instead augmentation factors were held constant throughout the potential trajectory space. This simplification was driven by the fact that ground to flight traceability and sting effects are not well understood. Given the sensitivity of the reaction control system jet heating augmentation to configuration, geometry, and orientation the focus in the present paper is on the methodology used to develop the models and the lessons learned from the data. The models that are outlined in the present work are specific to the aerothermal database used to design the thermal protection system for the Exploration Flight Test 1 vehicle.
\end{abstract}

\section{Nomenclature}

$\begin{array}{ll}\rho & \text { Density } \\ A F & \text { Augmentation Factor } \\ A o A & \text { Angle of Attack } \\ H R & \text { Enthalpy Ratio } \\ M R & \text { Momentum Ratio } \\ q & \text { Heat flux } \\ R e_{D} & \text { Reynolds number based on vehicle diameter } \\ \text { MR } & \text { Momentum ratio } \\ \mathrm{C}_{\mathrm{p}} & \text { Specific heat at constant pressure } \\ \mathrm{CFD} & \text { Computational Fluid Dynamics } \\ \mathrm{CM} & \text { Command module } \\ \mathrm{CUBRC} & \text { Calspan University of Buffalo Research Center } \\ \mathrm{D} & \text { Diameter } \\ \text { DES } & \text { Detached Eddy Simulation } \\ \text { EFT-1 } & \text { Exploration Flight Test 1 } \\ \mathrm{H} & \text { Total enthalpy } \\ \mathrm{HR} & \text { Enthalpy ratio }\end{array}$

\footnotetext{
*Research Scientist, Senior Member AIAA. Contact e-mail: Andrew.J.Hyatt@nasa.gov.

${ }^{\dagger}$ Aerospace Engineer, Applied Aerosciences and CFD Branch, Member AIAA.
} 


$\begin{array}{ll}\text { ISS } & \text { International Space Station } \\ \text { JI } & \text { Jet Interaction } \\ \text { LAS } & \text { Launch Abort System } \\ \text { LENS } & \text { Large Energy National Shock } \\ \text { LEO } & \text { Low Earth Orbit } \\ \text { LES } & \text { Large Eddy Simulation } \\ \text { MPCV } & \text { Multi-Purpose Crew Vehicle } \\ \text { NO } & \text { Nitric Oxide } \\ \text { OML } & \text { Outer Mold Line } \\ \text { PLIF } & \text { Planar Laser-Induced Fluorescence } \\ \text { RCS } & \text { Reaction Control System } \\ \text { RSI } & \text { Reusable Surface Insulation } \\ \text { TPS } & \text { Thermal Protection System } \\ \text { TSP } & \text { Temperature Sensitive Paint } \\ \text { V } & \text { Velocity } \\ \infty & \text { Freestream value } \\ \text { c } & \text { Value in the reaction control system jet chamber } \\ \text { cavity } & \text { Cavity induced } \\ \text { CFD, Turb } & \text { Reference value from turbulent CFD } \\ \text { Data } & \text { Reference value from experimental data } \\ \text { jet } & \text { Value at reaction control system jet exit } \\ \text { RCS } & \text { RCS specific (without specifying the reference source) } \\ \text { w } & \text { Value at the wall }\end{array}$

\section{Background}

The Orion Multi-Purpose Crew Vehicle (MPCV) is NASA's next generation human space exploration system. The geometry is similar to that of Apollo, though larger in diameter. ${ }^{1}$ It has been designed to carry up to four astronauts and is capable of Earth entries from beyond Low Earth Orbit (LEO).

The MPCV Aerosciences team was tasked with developing the aero/aerothermal environments database to be used for the design of the capsule. The aerothermal design database has been developed using engineering fidelity tools anchored to a large number of smooth Outer Mold Line (OML) Computational Fluid Dynamics (CFD) solutions, ${ }^{2}$ along with a series of augmentation factor (AF) models, or multipliers. The augmentation factor models combine with the smooth OML database to provide surface environments at all locations on the vehicle for the entire potential trajectory space. One example of an augmentation model is the Reaction Control System (RCS) model.

The MPCV includes an RCS which will be used to provide active control while flying guided lifting trajectories during atmospheric entry. The RCS is composed of 12 hydrazine $\left(\mathrm{N}_{2} \mathrm{H}_{4}\right)$ thrusters placed in pairs at six locations on the aft-body corresponding to roll left, roll right, yaw left, yaw right, pitch down, and pitch up (closest to the heat shield), as shown in Fig. 1. The two jets in each pair can be fired individual or in tandem, referred to as single and dual string respectively. When the RCS jets fire, the resulting plumes disrupt the flow-field causing significant changes to the surface environments. Experimental campaigns conducted during the Apollo program found augmentation levels as high as 4 for the yaw jets and as high as 11 for the forward facing roll jets, ${ }^{3}$ though baseline aft-body heating levels are generally much lower than those found on the heat-shield.

While the Apollo Command Module (CM) used an ablative Thermal Protection System (TPS) material on the aft-body, the MPCV CM has been designed to use a Reusable Surface Insulation (RSI) on the aft-body acreage, similar to that on the windward side of the Space Shuttle. The RSI materials, while lighter and more insulative (lower thermal conductivity), have a lower maximum heat flux capacity than the ablative TPS used on Apollo. The material selection made the design more sensitive to the peak heat rate, which on the aft-body were often driven by the RCS jet environments, making it one of the most critical aft-body augmentation models. 


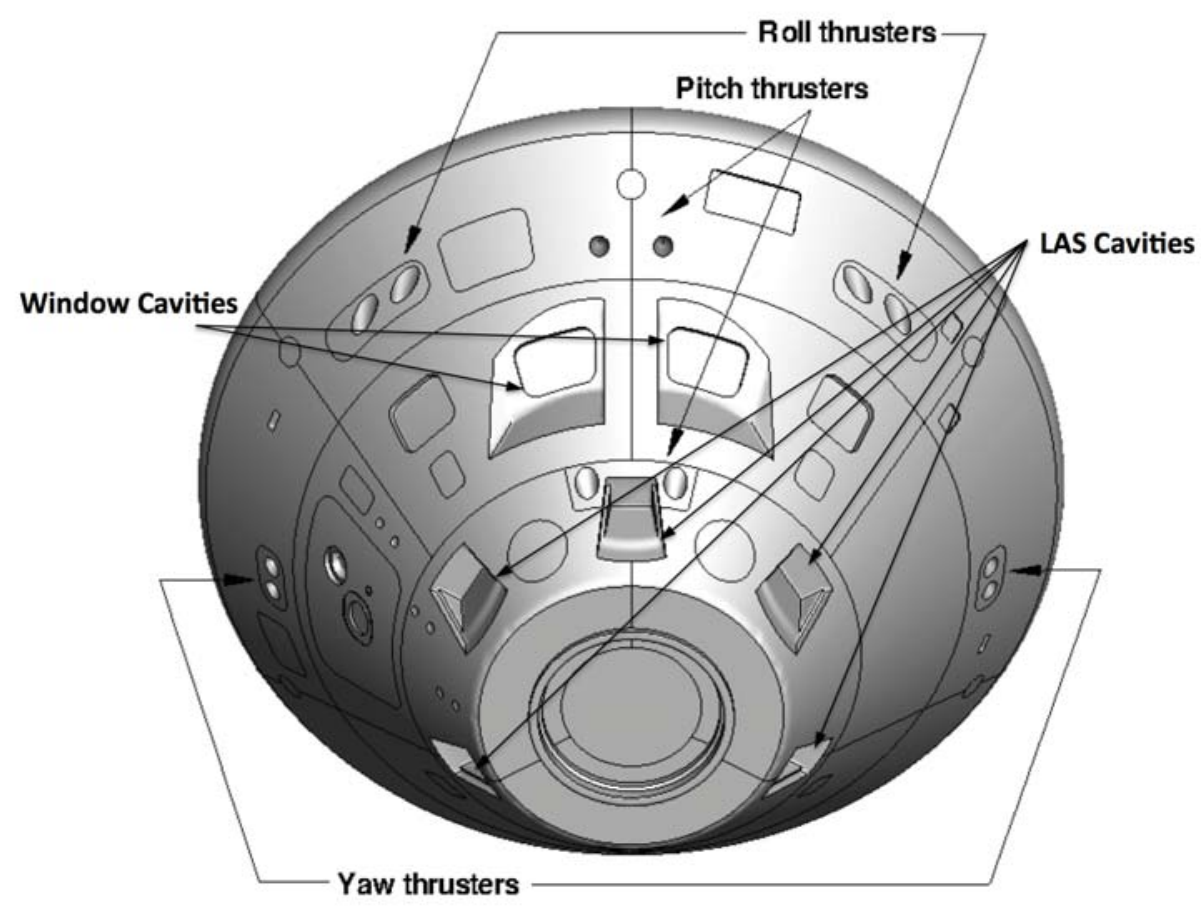

Figure 1. MPCV design layout with RCS (adapted from Dyakonov et. al. ${ }^{4}$ )

\section{Introduction}

Wake flows are difficult to model numerically, and modeling complexity increases when RCS jet plumes are introduced. Significant progress has been made in numerical simulation of wake flows behind blunt bodies. Comparisons of CFD predictions to Apollo aft-body flight data ${ }^{5,6}$ in the separated wake showed generally good agreement except when the flow became unsteady later in the trajectory. More recent computations using higher fidelity turbulence models, such as Detached Eddy Simulation (DES) and Large Eddy Simulation $(\mathrm{LES})^{7,8}$ showed better agreement with ground-based test data. However, these models are prohibitively expensive for routine production application.

A recent comparison of CFD predictions to ground-based experimental data of a MPCV-like model with RCS jets firing ${ }^{4}$ found as much as a factor of two discrepancy when the yaw jets were fired and multiple regions that were under-predicted by the CFD. While a number of investigations have been performed on jets into crossflow, ${ }^{9,10}$ those on jets into separated wake regions behind blunt bodies or capsules are more sparse, providing limited validation data for the advancement of CFD techniques for RCS jets in separated wake flows.

For the MPCV, with the exception of the yaw jets, all the RCS jets will remain in the separated region during the entire entry (see Fig. 2), and it is expected that the wake will be unsteady during much of the entry. It is also expected that the separation line will shift during the entry in the vicinity of the yaw jets, resulting in the yaw jets lying in both attached and separated flow regions during different portions of the entry. While in the attached region, the yaw jets produce similar flow phenomena to those described in previous investigations of jets into supersonic cross flow ${ }^{13}$ (see Fig. 3). A bow shock sets up in front of and wraps around the leading edge of the jet, which leads to high heating where the upstream separated recirculation zone draws the high enthalpy inviscid flow layer down toward the surface. When the roll and pitch down jets fire, the plumes penetrate and disrupt the shear layer, which promotes increased mixing between the high enthalpy post-shock inviscid flow and the separated wake, as shown in Fig. 4. In the case of the roll jets, the shear layer is close enough to the surface that the gross flow features are not dissimilar to that of jets into an attached crossflow. Similarly, they draw high enthalpy inviscid flow down toward the surface of the vehicle, resulting in particularly high augmentation factors given the baseline heating level in 
the separated wake region is relatively low.

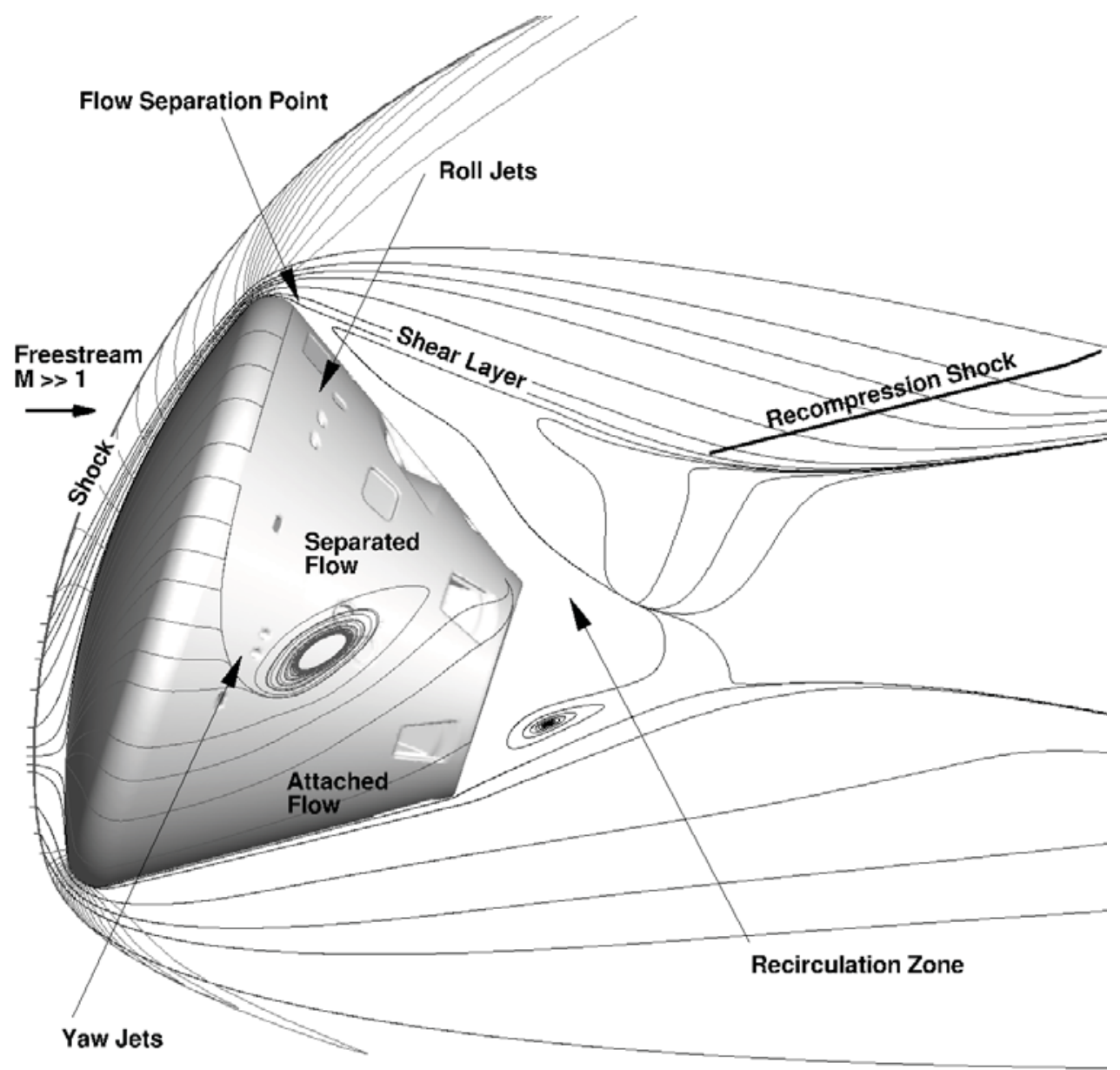

Figure 2. Schematic of the hypersonic flowfield with the RCS locations shown and CFD based shear lines providing insight into the separation line location (adapted from Dyakonov et al. $^{4}$ )

The Orion project and the development of the MPCV have brought a renewed interest in these flow-fields. A series of wind tunnel tests have been conducted on scale models with RCS firing. These tests have used both Temperature Sensitive Paint (TSP) as well as discrete instrumentation to measure surface heating. Additionally, techniques like nitric oxide (NO) Planar Laser-Induced Fluorescence ${ }^{11,12}$ (PLIF) have been used to visualize the flow-field. This new insight has helped shed light on the flow phenomena driving the RCS jet heating augmentation, which will prove useful to the development of numerical modeling techniques. However, until the new data can be used to improve the numerical modeling capability, the development of the RCS jet models must rely almost exclusively on the surface data gathered during recent ground based test campaigns.

The present paper has three primary objectives. The first is the compilation and distillation of the wind tunnel data acquired during the development of the RCS jet heating augmentation factor model development. The second is to identify the lessons learned from the data. And finally, the third is to describe the application of the newly developed augmentation factor models. These models are potentially conservative in their application of the new wind tunnel data in that they do not utilize correlations with potential similarity parameters. Instead the models apply constant augmentation factors, which bound all of the data, along the entire entry trajectory. This was done because of uncertainty in the correlations themselves due to limited perturbation of all potential similarity / scaling parameters, combined with uncertainty in flight traceability of the correlations and the potential influence of the sting in the data.

Finally, the development of the RCS models has been an on-going process as new data have become 


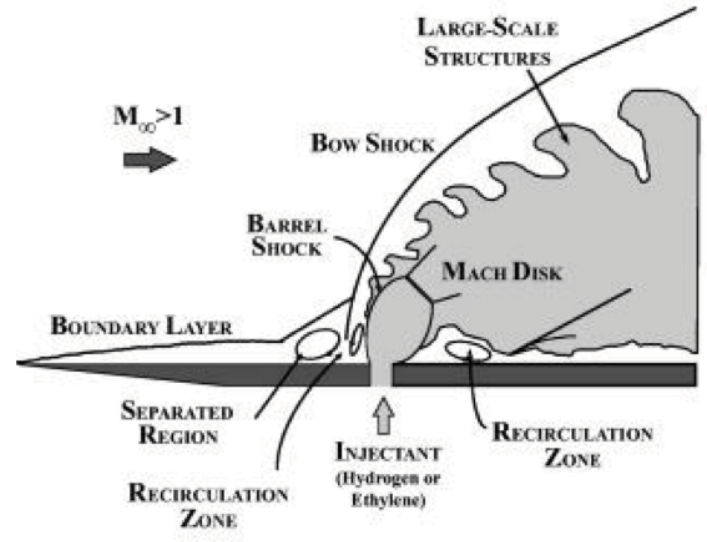

(a) Instantaneous side view at the centerline axis of the jet

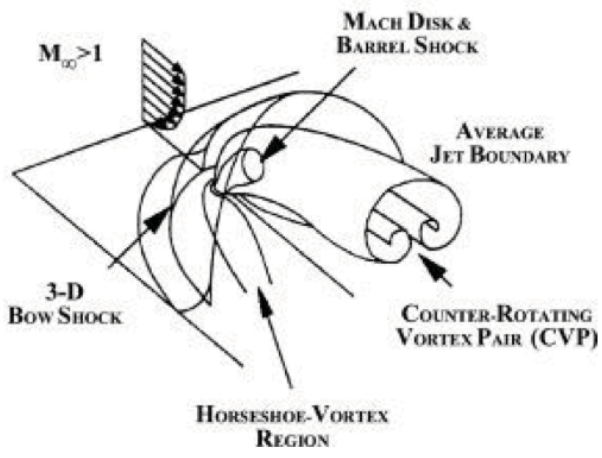

(b) 3D perspective of the averaged features of the flowfield

Figure 3. Schematic of an under-expanded transverse jet into a supersonic crossflow. (from Gruber et al. ${ }^{14}$ )

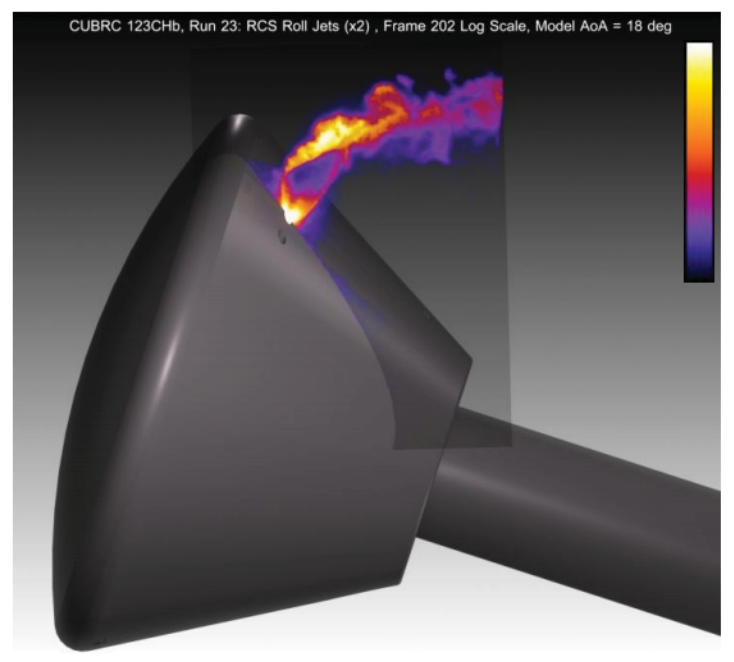

(a) $5743.3 \mathrm{kPa}(833 \mathrm{psia}) \mathrm{RCS}$ roll jet pair issuing into a $0.689 \mathrm{kPa}(0.1 \mathrm{psia})$ Mach 8 flowfield

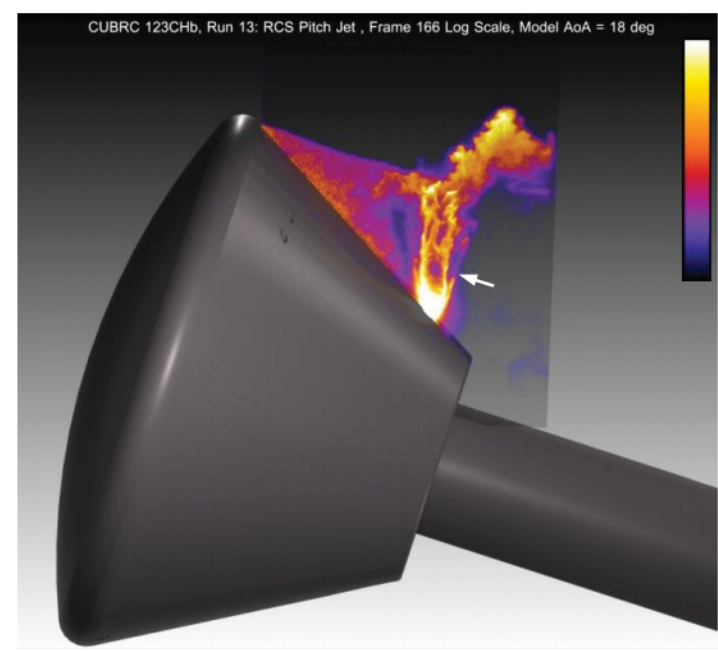

(b) $6053.6 \mathrm{kPa}(878$ psia) pitch down jet issuing into a $8.27 \mathrm{kPa}$ (1.2 psia) Mach 8 flowfield

Figure 4. ViDI rendering of the capsule model at $18^{\circ}$ AoA with NO PLIF visualization (from Combs et al. ${ }^{12}$ )

available and as the design of the vehicle has matured. The models detailed here are those used for the design of the first test flight of the vehicle, Exploration Flight Test 1 (EFT-1), currently scheduled for Fall of 2014 .

\section{Testing}

A number of ground tests were conducted to gather the data used to develop the RCS jet models. These tests used both discrete instrumentation as well as global surface imagery, with TSP techniques. In some cases, flow-field imagery using Schlieren or PLIF techniques were also employed. These ground tests were performed in multiple facilities. The test conditions were developed based on the potential entry corridor, which was defined by the early design trajectories. These trajectories included both lunar skip and direct entries as well as returns from the International Space Station (ISS), the corresponding velocity-altitude space is shown in Fig. 5. The target freestream conditions for design space points in the velocity-altitude 
map are listed in Table 1.

For testing representative RCS jet flow-fields it was also critical to match the appropriate RCS jet-tofreestream conditions. While the optimal jet-to-freestream similarity parameters have not been established, several potential parameters were explored to help insure that the models would not under-predict the flight conditions, including the momentum-ratio (MR), and enthalpy-ratio (HR), which are defined as:

$$
\begin{gathered}
M R=\frac{\rho_{\text {jet }} V_{\text {jet }}^{2} A_{\text {jet }}}{\rho_{\infty} V_{\infty}^{2}\left(\pi D_{\text {model }}^{2} / 4\right)} \\
H R=\frac{H_{\text {jet }}}{H_{\infty}}=\frac{C_{p_{j e t} T_{j e t}+V_{\text {jet }}^{2} / 2}}{C_{p_{N_{2}}} T_{\infty}+V_{\infty}^{2} / 2}
\end{gathered}
$$

The rational behind these parameters was as follows. As the RCS jet exits the nozzle, the plume begins to interact with the surrounding flow-field. The heating augmentation as a result of the RCS jets can be caused by: (1) increased mixing across the shear layer, (2) the plumes redirecting the high enthalpy inviscid flow toward the surface, or (3) the plumes themselves depositing energy on the surface. Depending on the orientation of the jet, the plume is deflected by and begins mixing with the high enthalpy post shock inviscid flow layer. How quickly the plume is deflected and diffused is tied to the relative momentum of the jet as compared to the freestream. This is particularly true of jets in attached flow region and those in locations where the shear layer is close to the surface, like the roll RCS jets. The heating augmentation around RCS jets that are deep in the subsonic wake region, like the pitch down RCS jets, are likely dependent on the relative expansion of the plumes at the nozzle exit. Given that the jets are relatively under-expanded during the entire heating pulse, the momentum ratio can be used to capture this phenomena as well.

The enthalpy ratio helps characterize the relative energy contribution from the jet as compared to the surrounding flow-field to the heating augmentation. Flow phenomena leading to heating augmentation driven primarily by the redirection of the high enthalpy inviscid flow were expected to correlate less with enthalpy ratio. On the other hand, flow phenomena leading to heating augmentation driven by the plume mixing with the wake and in turn depositing energy on the surface were expected to correlate more with the enthalpy ratio.

Equations (1) and (2) were then used to determine the value of the similarity parameters at the target flight conditions using the provided RCS jet exit conditions of $\mathrm{T}_{\text {jet }} \approx 287 \mathrm{~K}, \mathrm{p}_{\text {jet }} \approx 3.8 \mathrm{kPa}$, and $\mathrm{M} \approx 4.6$. The values of MR and HR at each target flight condition have also been included in Table 1. Once the flight values of the similarity parameters were established they could be matched at the tested freestream conditions by varying the jet chamber conditions. Four wind tunnel tests were used directly in the development of the RCS jet heating augmentation factor model, utilizing two different facilities and both discrete and global surface measurement techniques. The bounds of the relevant parameter space for each test are given in Table 2 .

Table 1. Target freestream conditions (adapted from Dyakonov, et al. ${ }^{4}$ )

\begin{tabular}{|c|c|c|c|c|c||c|c|}
\hline Mach & $V_{\infty}, \mathbf{~ k m} / \mathbf{s}$ & $\begin{array}{c}\rho_{\infty}, \mathbf{k g} / \mathbf{m}^{3} \\
\times \mathbf{1 0}^{3}\end{array}$ & $T_{\infty}, \mathbf{K}$ & $\begin{array}{c}\mathbf{R e}_{D} \\
\times \mathbf{1 0}^{-6}\end{array}$ & $\mathbf{A o A}, \mathbf{d e g}$. & $\begin{array}{c}\mathbf{M R} \\
\times \mathbf{1 0}^{3}\end{array}$ & $\mathbf{H R}$ \\
\hline \hline 33.9 & 10.50 & 0.2173 & 239.0 & 0.2724 & 18 & 1.4 & $<0.1$ \\
\hline 31.2 & 9.83 & 0.3173 & 247.3 & 0.3767 & 18 & 1.1 & $<0.1$ \\
\hline 25.9 & 8.00 & 0.1974 & 237.0 & 0.1880 & 18 & 2.7 & 0.10 \\
\hline 21.1 & 6.60 & 0.2837 & 244.8 & 0.2254 & 18 & 2.8 & 0.14 \\
\hline 18.4 & 6.00 & 0.6782 & 264.6 & 0.5001 & 18 & 1.4 & 0.17 \\
\hline 15.2 & 5.00 & 1.465 & 270.4 & 0.9042 & 18 & 0.9 & 0.25 \\
\hline 12.4 & 4.00 & 2.426 & 260.2 & 1.188 & 18 & 0.9 & 0.39 \\
\hline 6.43 & 2.00 & 6.852 & 240.6 & 1.640 & 18 & 1.3 & 1.54 \\
\hline
\end{tabular}




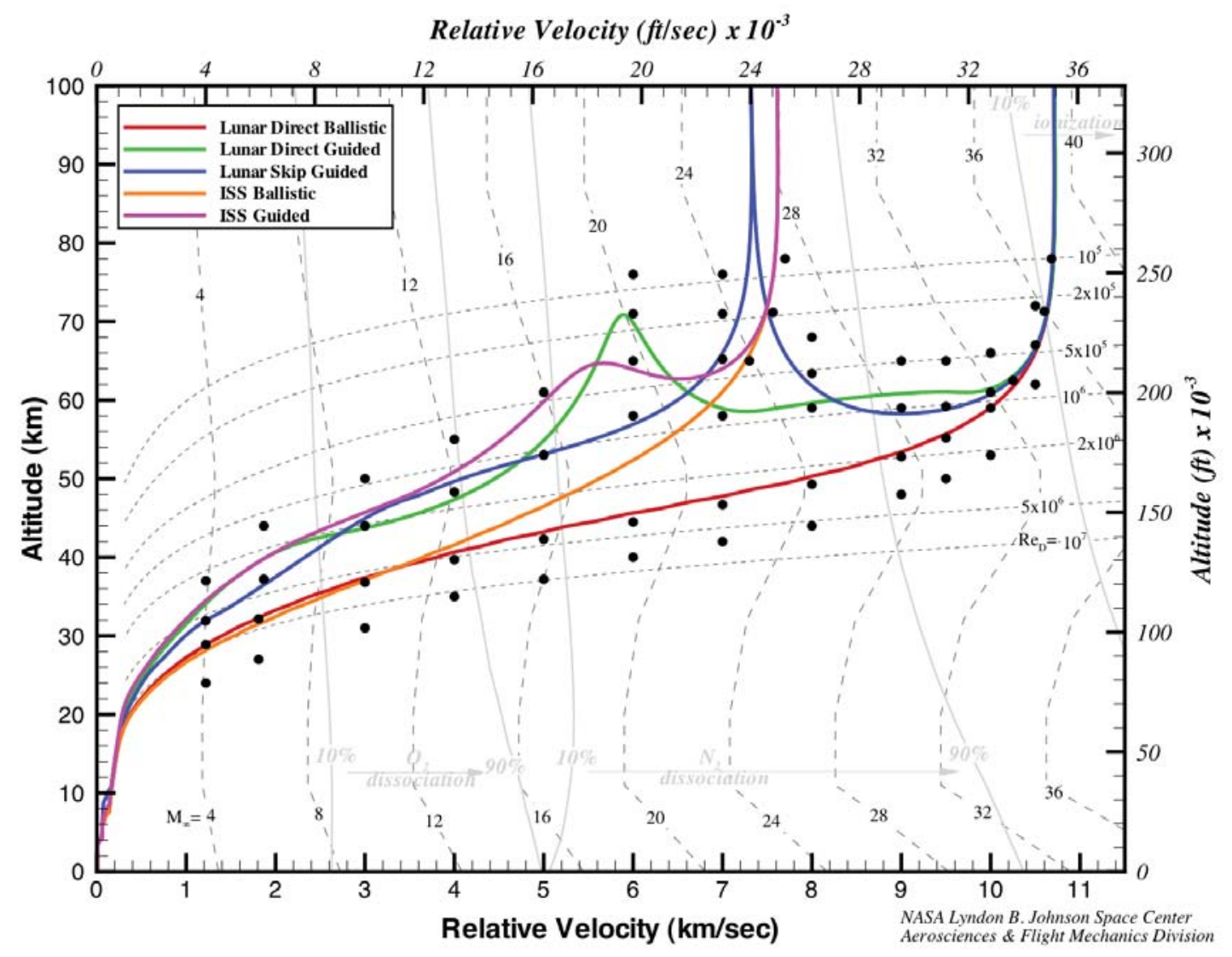

Figure 5. Entry corridor in Velocity-Altitude space, where the closed symbols identify points at which detailed flow calculations have been performed. (from Greathouse et. al. ${ }^{1}$ )

\section{A. $63-\mathrm{CH}$}

The 63-CH test was the second of a series of two pathfinder tests for MPCV aft-body RCS jet augmentation environments. The two pathfinder tests were very similar with the main difference being the maturity of the vehicle design. The first test, described in Buck, et al., ${ }^{16}$ tested RCS jet influences on a scaled Apollo vehicle as well as an early concept scaled MPCV vehicle. The 63-CH test itself was the first RCS jet test performed on a matured MPCV configuration. ${ }^{17}$ Both tests were conducted in the Langley 31-inch Mach 10 tunnel and utilized TSP to capture global surface heat flux levels. The data from two runs, one with no RCS jets firing and a second with the RCS jets firing, were compared to identify the RCS jet region of influence, as seen in Fig. 6, which was then used to drive instrumentation placement on future tests. The 63-CH test played a crucial role in determining the optimal instrumentation placement in subsequent tests using discrete instrumentation. 
Table 2. Wind tunnel testing parameter space for each of the four test programs.

\begin{tabular}{|c|c|c|c|c|c|c|c|c|}
\hline \multirow{2}{*}{ Parameter } & \multicolumn{2}{|c|}{ 63-CH } & \multicolumn{2}{|c|}{ 66B-CH } & \multicolumn{2}{|c|}{ 95-CH } & \multicolumn{2}{|c|}{$123-\mathrm{CH}$} \\
\hline & Min & Max & Min & Max & Min & Max & Min & Max \\
\hline Facility & \multicolumn{2}{|c|}{ LaRC Mach 10} & \multicolumn{2}{|c|}{ CUBRC LENS I } & \multicolumn{2}{|c|}{ LaRC Mach 10} & \multicolumn{2}{|c|}{ CUBRC LENS I } \\
\hline Flow medium & \multicolumn{2}{|c|}{ Air } & \multicolumn{2}{|c|}{$\mathrm{N}_{2}$} & \multicolumn{2}{|c|}{ Air } & \multicolumn{2}{|c|}{$\mathrm{N}_{2}$ and Air } \\
\hline Model scale & \multicolumn{2}{|c|}{0.025} & \multicolumn{2}{|c|}{0.0505} & \multicolumn{2}{|c|}{0.025} & \multicolumn{2}{|c|}{0.0505} \\
\hline Aft-body cone angle, deg. & \multicolumn{2}{|c|}{32.5} & \multicolumn{2}{|c|}{32.5} & \multicolumn{2}{|c|}{32.5 and 30.0} & \multicolumn{2}{|c|}{32.5 and 30.0} \\
\hline Jet effluent gas & \multicolumn{2}{|c|}{ Air } & \multicolumn{2}{|c|}{$\mathrm{H}_{2} / \mathrm{N}_{2}(55 \% / 45 \%)$} & \multicolumn{2}{|c|}{ Air } & \multicolumn{2}{|c|}{$\mathrm{H}_{2} / \mathrm{N}_{2}(55 \% / 45 \%)^{*}$} \\
\hline AoA, deg. & 18.0 & 28.0 & 15.5 & 28.0 & 15.5 & 28.0 & 13.0 & 22.0 \\
\hline Mach & $\approx 10$ & $\approx 10$ & $\approx 8$ & $\approx 8$ & $\approx 10$ & $\approx 10$ & $\approx 8$ & $\approx 8$ \\
\hline $\operatorname{Re}_{\mathrm{D}} \times 10^{-6}$ & $\approx 0.21$ & $\approx 0.73$ & $\approx 1.0$ & $\approx 20.0$ & $\approx 0.26$ & $\approx 0.73$ & $\approx 2.0$ & $\approx 15.0$ \\
\hline Enthalpy, MJ/kg & $\approx 1.0$ & $\approx 1.0$ & $\approx 1.0$ & $\approx 3.0$ & $\approx 1.0$ & $\approx 1.0$ & $\approx 1.0$ & $\approx 1.0$ \\
\hline $\mathrm{MR} \times 10^{3}$ & $\approx 0.12$ & $\approx 1.4$ & $\approx 0.3$ & $\approx 2.2$ & $\approx 0.9$ & $\approx 2.2$ & $\approx 0.58$ & $\approx 2.2$ \\
\hline $\mathrm{HR}$ & $\approx 0.30$ & $\approx 0.30$ & $\approx 0.33$ & $\approx 0.90$ & $\approx 0.30$ & $\approx 0.35$ & 0.53 & 0.85 \\
\hline RCS jet banks studied & \multicolumn{2}{|c|}{ Roll, PD, Yaw } & \multicolumn{2}{|c|}{ Roll, PD, PU, Yaw } & \multicolumn{2}{|c|}{ Roll, PD, Yaw } & \multicolumn{2}{|c|}{ Roll, PD } \\
\hline Combinations & & & \multicolumn{2}{|c|}{ Roll / PD, Roll / PU } & & & \multicolumn{2}{|c|}{ Roll / PD } \\
\hline Single string & & & \multicolumn{2}{|c|}{ Roll, PD, PU, Yaw } & \multirow{2}{*}{\multicolumn{2}{|c|}{ Roll, PD, Yaw }} & \multicolumn{2}{|c|}{ Roll, PD, PU, Yaw } \\
\hline Dual string & \multicolumn{2}{|c|}{ Roll, PD, Yaw } & \multicolumn{2}{|c|}{ Roll, PD, PU, Yaw } & & & & $\mathrm{PD}$ \\
\hline Data Remarks & $\begin{array}{l}\text { Globa } \\
\text { Qual }\end{array}$ & $\begin{array}{l}\text { (TSP) } \\
\text { ative }\end{array}$ & $\begin{array}{r}\approx 40 \\
\mathrm{~T} \\
\mathrm{Qu}\end{array}$ & $\begin{array}{l}\text { iscrete } \\
\text { films } \\
\text { tative }\end{array}$ & $\begin{array}{r}\text { Glob } \\
\text { Qu } \\
\text { Compa } \\
\text { Qua }\end{array}$ & $\begin{array}{l}(\mathrm{TSP}) \\
\text { ative } \\
\text { Geometry } \\
\text { tative }\end{array}$ & $\begin{array}{r}>30 \\
\mathrm{~T} \\
\mathrm{Qu}\end{array}$ & $\begin{array}{l}\text { iscrete } \\
\text { films } \\
\text { tative }\end{array}$ \\
\hline
\end{tabular}

*Note: A small percentage of $\mathrm{NO}$ was added to some runs to seed the flow to improve the NO-PLIF visualization

\section{B. $66 \mathrm{~B}-\mathrm{CH}$}

The 66B-CH test was performed in the Calspan University of Buffalo Research Center (CUBRC) Large Energy National Shock (LENS) facility tunnel I. ${ }^{18}$ The focus of this test was on both the RCS and the cavity influence to the aft-body surface heat flux. The test article was a 0.0505 scale model, heavily instrumented with over 400 discrete thin film sensors, which were strategically placed to capture the critical RCS and cavity influences, Fig. 7. As mentioned earlier, the placement of these sensors with respect to RCS was based largely on the results of previous TSP data from 63-CH. One roll bank, one yaw bank (those on the left side of Fig. 7) and both the pitch down and pitch up banks were all plumbed such that each could be fired independently or in combination. The jets on the left side were selected based on the placement of the hatch and ground support hand holds being located on that side. Single string and dual string firings from any RCS bank could also be accommodated. The test was also intended to validate cavity heating models, which led to a number of instruments being placed in and around cavity

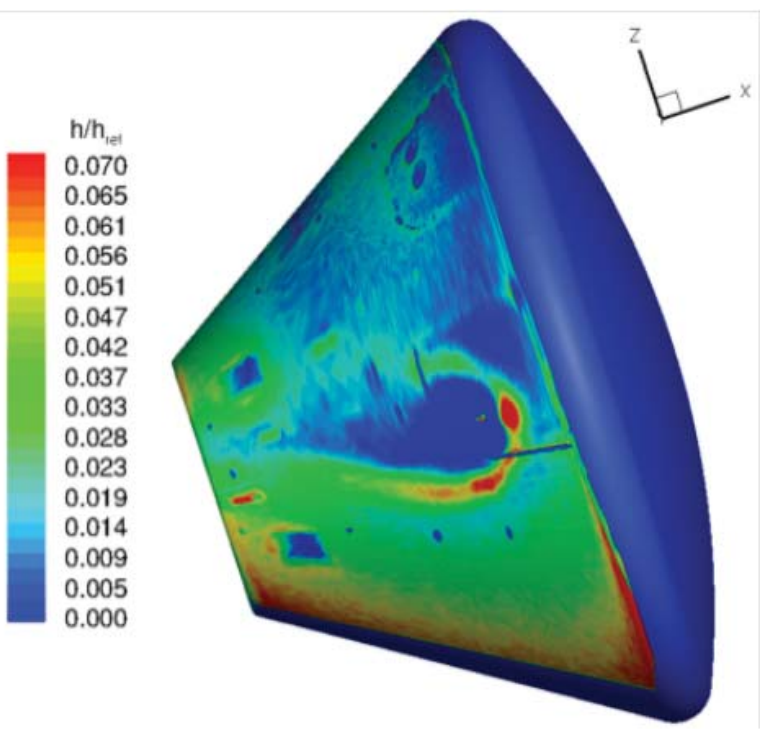

Figure 6. Representative TSP heating data with dual yaw RCS jets firing (from Dyakonov et al. $^{4}$ )

features. Additionally, instrumented covers were designed for the four largest cavity features in the separated region, the two Launch Abort System (LAS) cavities and the large window cavities, to be used to measure the baseline (smooth) gauge level in the calculation of cavity augmentation factors. Several runs 
were performed with the RCS firing while the covers were in place. These data were useful in evaluating the combined cavity and RCS environments. An array of sensors were placed along the windside centerline, which were used to compare to CFD solutions to determine the state of the boundary layer at each freestream condition, laminar, turbulent or transitional, on the attached aft-body. Regions without any cavity features and not expected to be strongly influenced by RCS jet heating augmentation were left un-instrumented.

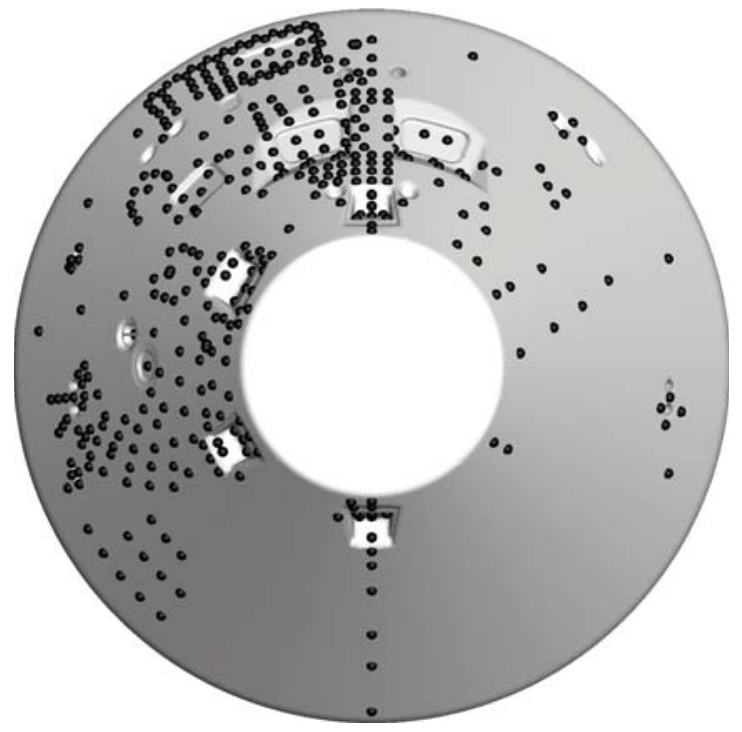

(a) With window and LAS cavities exposed

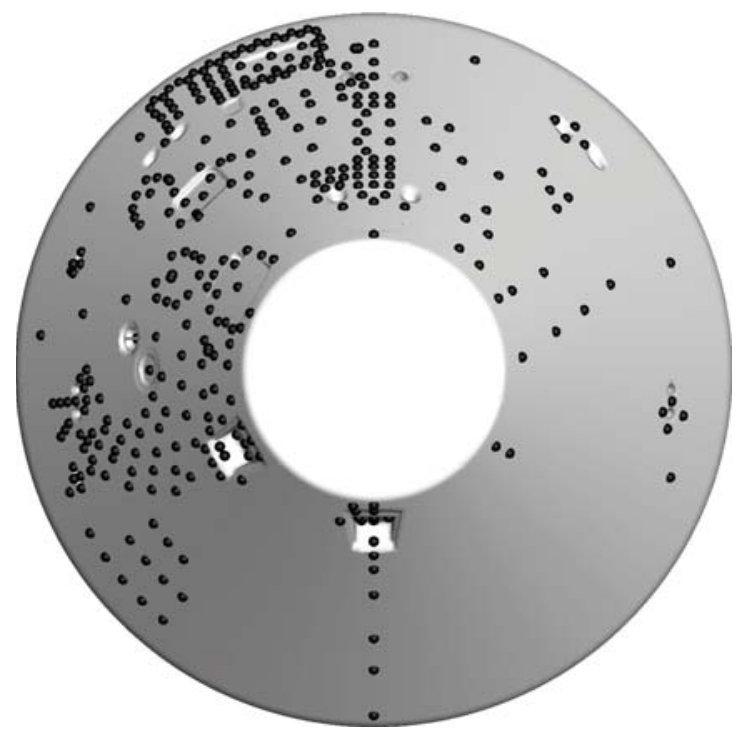

(b) With window and LAS cavities covered

Figure 7. 66B-CH instrumentation layout

The test explored a long list of parameters. Several freestream parameters were explored including Reynolds number, freestream enthalpy, and angle of attack. The potential jet-to-freestream similarity parameters MR and HR were also explored. The test evaluated the influence of each of these parameters with each RCS bank (roll, pitch down, pitch up and yaw) and combinations of roll with pitch down/up to the extent possible within the schedule and budget limitations. The test also evaluated the influence of $\mathrm{Re}_{\mathrm{D}}$ and AoA on all of the cavity features on the aft-body. It was not possible to evaluate each parameter $f$ ully for each RCS jet bank. This led to the prioritization of each of the RCS jet banks. Lower priority parameters at lower priority RCS jet banks were simply bounded.

The roll RCS jets were the highest priority, due to fact that they would be used the most and because they had the highest augmentation factors. The pitch down RCS were considered the next highest priority because the resulting heating augmentation was directly over the large window features on the aft-body as well as the variability of the heating augmentation with freestream and potential jet-to-freestream parameters. The yaw RCS jets were relatively low in priority because there were fewer aft-body features they would impact. The pitch up RCS jets were the lowest priority due to their relatively minor heating augmentation. Additionally, it was understood at the time that dual string firings were the default. This led to a strong bias in the testing toward dual string RCS jet firings.

Several of the freestream conditions were selected by attempting to match flight Reynolds numbers at critical trajectory points like peak dynamic pressure. The jet chamber conditions were determined by matching the flight MR and HR at the selected freestream trajectory points.

\section{C. $95-\mathrm{CH}$}

After the 66B-CH test was started, the MPCV program determined that the aft shell cone angle would have to be changed as the design of other systems became more mature. The geometry and configuration of the LAS cavities and the large window cavities were also changed. The 95- $\mathrm{CH}$ test was developed to quantify the changes in the heating augmentation due to changes in the configuration. The test was conducted in the Langley 31-in Mach 10 tunnel, again with TSP as the primary source of data. Two models were built, one that matched the 66B-CH model, and a second that match the latest flight configuration. These models 
were then tested at similar conditions to help characterize differences in the environments between the two geometries.

\section{D. $123-\mathrm{CH}$}

The 123-CH test was conducted in the CUBRC LENS-I reflected shock tunnel. The test was conducted in two parts.

The first part, designated as Part A, utilized the same model from the 66B-CH test and was intended to further explore several test parameters investigated during the 66B-CH test. In particular, the range of angle of attack was expanded, two intermediate $R e_{D}$ values were tested, and roll with pitch down RCS jet combinations were further investigated.

The second part, designated as Part B, used a new model with the updated backshell angle and a more uniform instrumentation layout, though with more resolution in several regions of interest based on the previous tests, as shown in Fig. 8. These regions included the roll RCS peak augmentation region, the walls of a pitch down nozzle and the separated wake reattachment location along the apex shoulder. Additionally, the model did not include any of the other backshell cavity features with the exception of the windside centerline Launch Abort

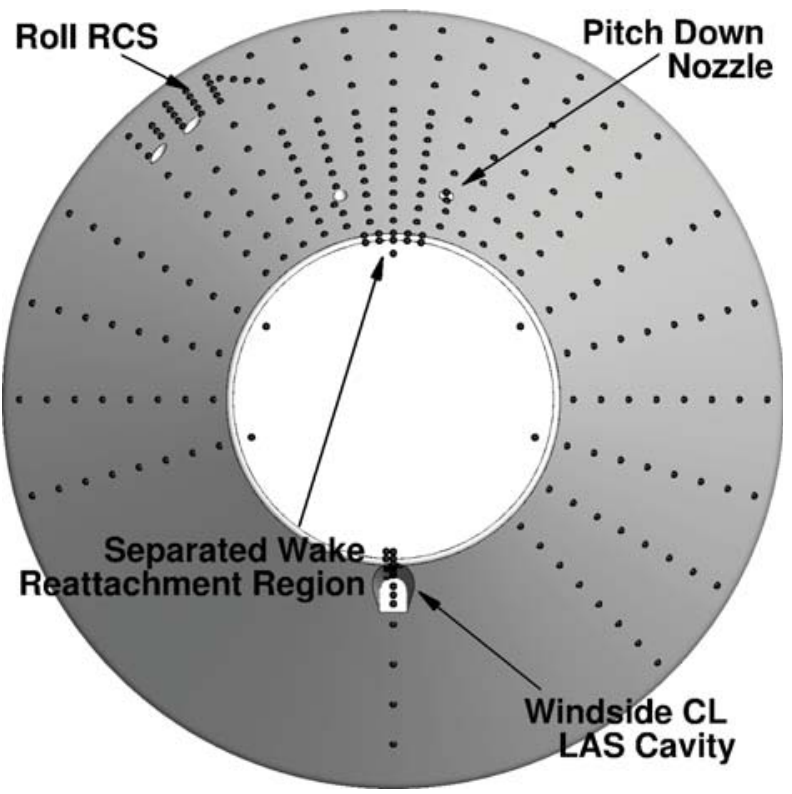

Figure 8. 123-CH part $\mathrm{B}$ instrumentation layout System (LAS) cavity. All of the LAS cavities had been redesigned but the windside centerline location was considered the most critical with respect to aerothermal environments.

The primary objectives of the test were to provide data to refine the models to accommodate the backshell angle change and LAS cavity redesign. Secondary objectives were to provide data to help validate numerical modeling tools for separated wake regions and RCS environments. In addition to surface instrumentation, the test used NO-PLIF techniques to visualize the interaction of the RCS jet with the wake flow, described in Combs et al. ${ }^{12}$

\section{Results}

The primary quantity of interest with each of these tests was the RCS jet heating augmentation factor. By calculating the ratio of heat flux from two test runs, with and without the RCS firing, the associated RCS jet augmentation factor was determined.

$$
A F_{\text {Data }}=\frac{q_{w}, R C S-\text { on, data }}{q_{w, R C S-o f f, \text { data }}}
$$

This ratio was evaluated for every gauge with data from two runs at similar freestream conditions, shown in Fig. 9. Peak augmentation factors of 13.0, 7.6, 2.8 and 5.8 were calculated for the roll, pitch down, pitch up and yaw RCS jets respectively. A series of augmentation factor plots were then compared to find trends with various potential similarity parameters.

\section{A. Momentum Ratio Trends}

The jet-to-freestream momentum ratio was one of the potential similarity parameters that was evaluated. Figure 10 shows the augmentation factors resulting from dual roll RCS firing while varying the momentum ratio, increasing from top left to bottom right. In general, the roll RCS jet heating augmentation swept from just forward of the nozzles, inboard and back towards the apex. The peak augmentation was seen just upstream and inboard of the nozzle exit where the plume redirected high enthalpy post shock inviscid flow toward the surface. Similar peaks where seen for other RCS jet banks as well and the spatial region 


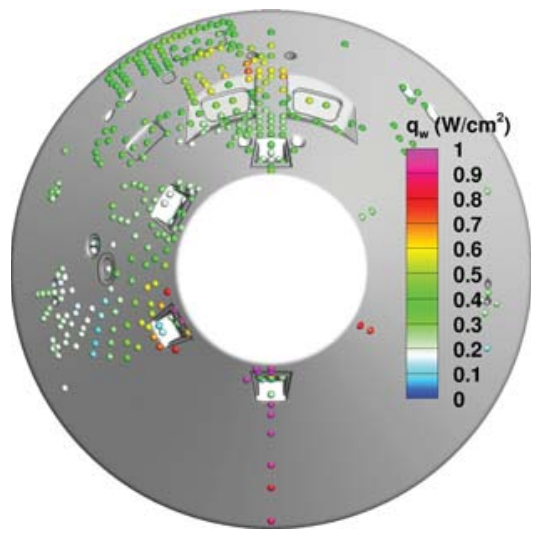

(a) Heat flux, no RCS jets firing

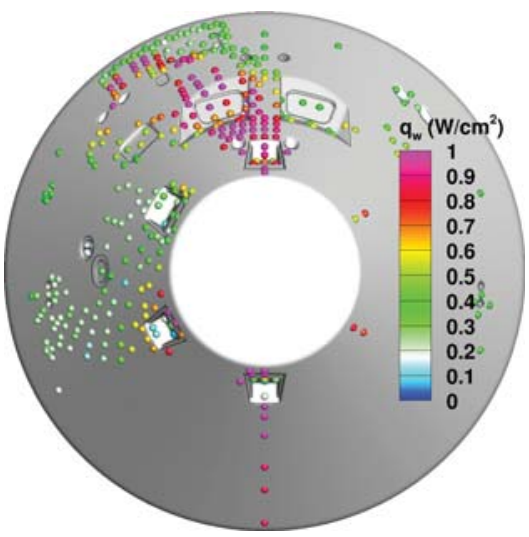

(b) Heat flux, dual roll RCS jets firing

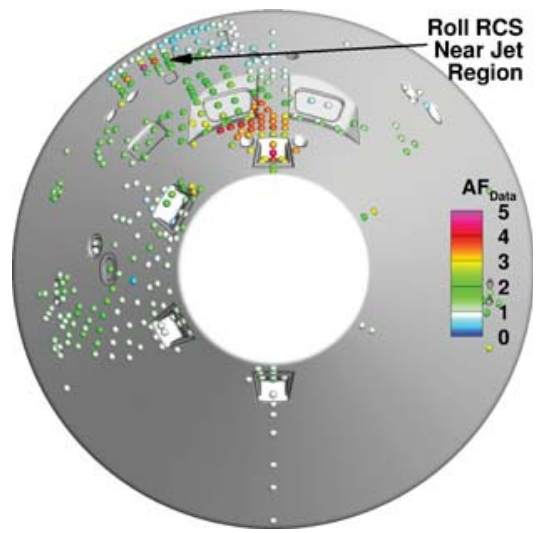

(c) Calculated $\mathrm{AF}_{\text {Data }}$

Figure 9. An example of scatter data from $66 \mathrm{~B}-\mathrm{CH}$ and computed augmentation factors $\left(\mathrm{AF}_{\mathrm{Data}}\right)$.

containing these peaks became known as the near jet region. The augmentation factors in the near jet region between the roll jet nozzles and the heat shield shoulder increased with increasing momentum ratio. Additionally, the augmentation factors in the large window features and between the large window features and the apex tended to decrease with increasing momentum ratio. The variation of the pitch down RCS augmentation factors with momentum ratio are shown in Fig. 11, increasing momentum ratio from top left to bottom right. The augmentation factors in the region between the two pitch down nozzles and the large window features increased with increasing momentum ratio. The location of the peak augmentation factor shifted though. At low MR the peak was directly in front of the nozzles. As the MR was increased (higher $\mathrm{p}_{c}$ ) the nozzles became more under-expanded, resulting in increased interaction between the two plumes. This interaction shifted the peak augmentation factor from directly in front of the nozzles to between them. A trend with momentum ratio was less evident for the yaw RCS, shown in Fig. 12. While the highest augmentation factors are seen at the highest momentum ratio, the lowest augmentation factors are seen with the intermediate momentum ratio rather than the lowest tested. The pitch up nozzles were not tested enough to evaluate trends with momentum ratio.

The scatter plots helped identify general trends but made it difficult to develop quantitative conclusions. Figure 13 shows the augmentation factor in the near jet roll region as a function of momentum ratio. The plot includes all dual roll RCS jet firing runs from tests 66B-CH (blue), 95-CH (green), and 123-CH (orange). The 95-CH TSP data have been interpolated to either the 66B-CH or 123-CH gauge locations depending on the geometry that was tested. The heat flux at an aft-body windside location (shown in black) is also shown as a function of momentum ratio along a reference EFT-1 trajectory. Each column of augmentation factor values corespsond to the gauge locations shown in the upper right for a given run. A clear trend between the "peak" augmentation factor in this region with the momentum ratio can been seen. Additionally, it can be seen that the data suggest the peak augmentation factor would not occur at peak heating on this particular trajectory.

\section{B. Enthalpy Ratio Trends}

The jet-to-freestream enthalpy ratio was another potential similarity parameter that was investigated. The data showed the augmentation factors were far less dependent on the enthalpy ratio than the momentum ratio. Figure 14 shows augmentation factor due to dual roll RCS jet firing while varying enthalpy ratio, increasing from top left to bottom right. In the near jet region, the highest augmentation factors were seen at the highest enthalpy ratio and qualitatively they appeared to decrease as the enthalpy ratio was decreased. However, the peak measured augmentation factor at the lowest HR, Fig. 14a, was greater than the peak seen in Fig. 14b, making it difficult to characterize a trend conclusively. One possible explanation was that the true peaks were not always captured by the discrete instrumentation. Another possibility was that the small variation in MR overwhelmed the influence of the HR. Figures 14c and 14d had slightly higher momentum ratios than those in Figs. 14a and 14b. In the far-field, the heating augmentation was assumed to 


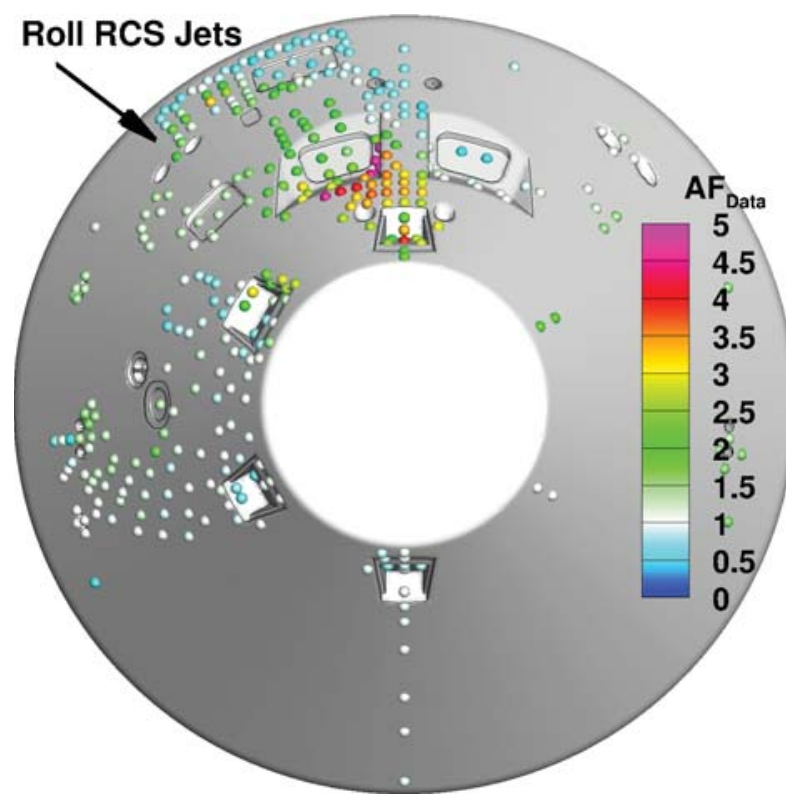

(a) $\mathrm{MR}=0.68 \times 10^{-3}$

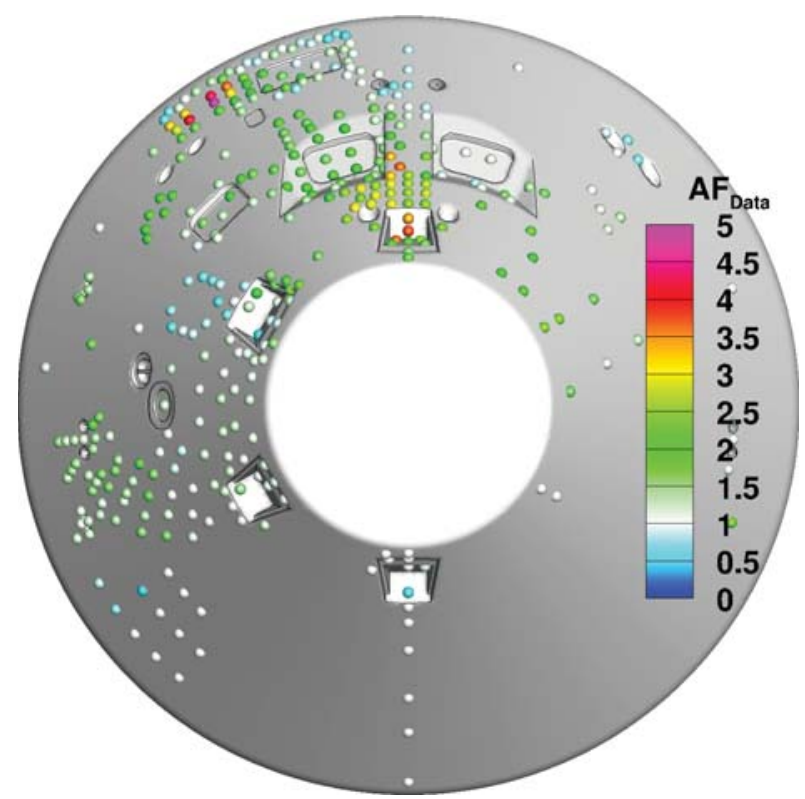

(c) $\mathrm{MR}=1.6 \times 10^{-3}$

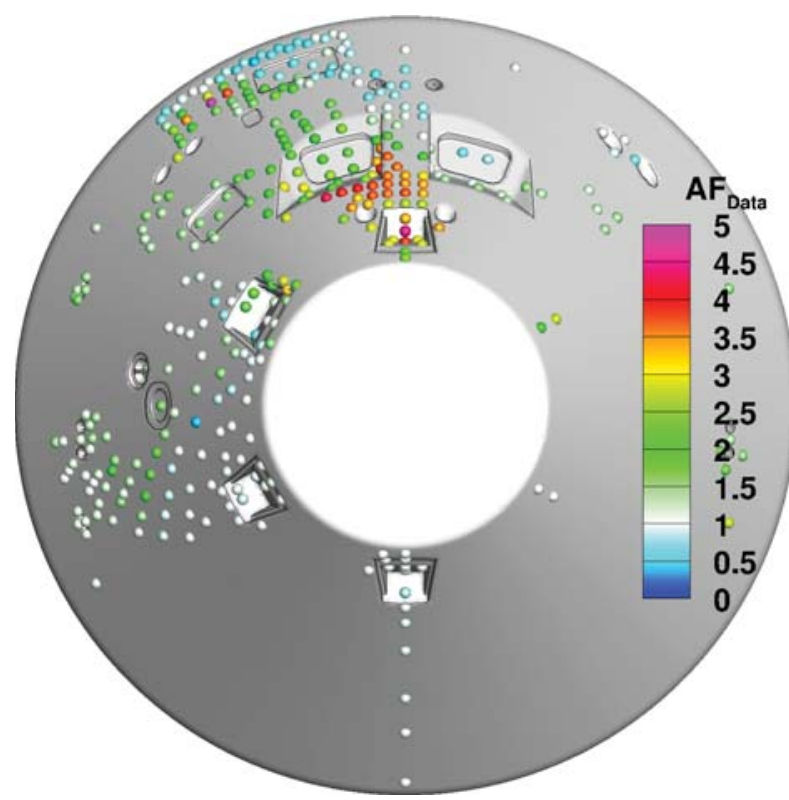

(b) $\mathrm{MR}=0.84 \times 10^{-3}$

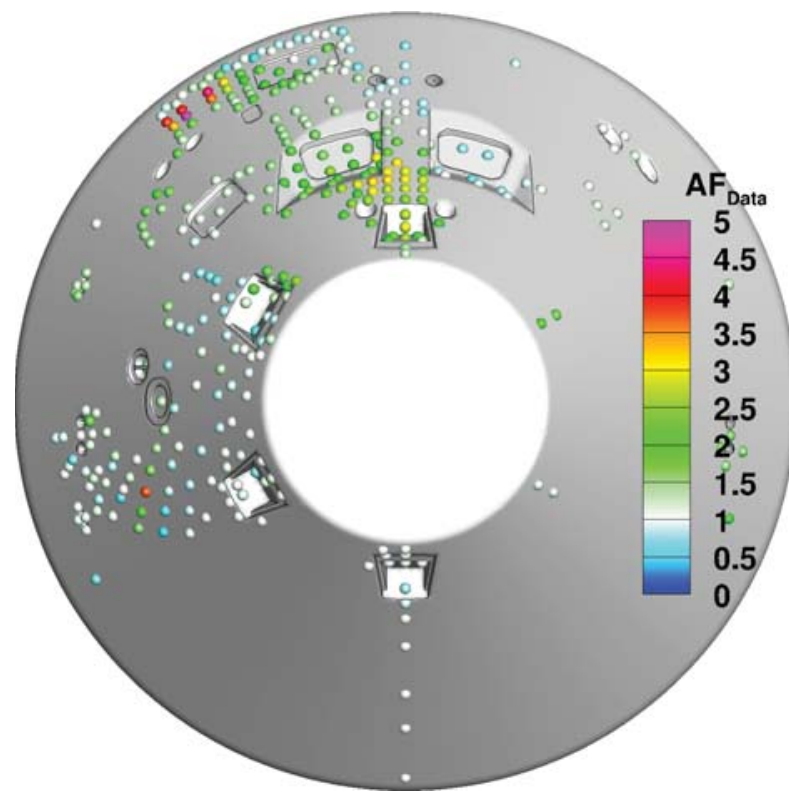

(d) $\mathrm{MR}=1.9 \times 10^{-3}$

Figure 10. Scatter plot of augmentation factor while firing dual roll RCS jets at $\mathbf{M}_{\infty} \approx \mathbf{8 . 0}, \mathbf{R e}_{\mathrm{D}} \approx \mathbf{2 . 0} \times 10^{6}$, $\mathrm{AoA}=18.0, \mathrm{HR} \approx 0.55$, while varying momentum ratio

be primarily driven by the plume disrupting the shear layer, leading to increased mixing of the high enthalpy inviscid flow with the wake. With this flow phenomena, there was limited entrainment of the plume in the wake, as seen in Fig. 4a, which suggested the enthalpy ratio should have limited influence. The data in the far-field showed limited dependence on the enthalpy ratio as expected. The highest augmentation factors in the far-field were seen at the lowest enthalpy ratio. As with the near jet region, the increased heating seen at the lowest enthalpy ratio may have been tied more to the momentum ratio. Variation of the enthalpy ratio with other RCS jet banks was limited due to budget and schedule constraints. 


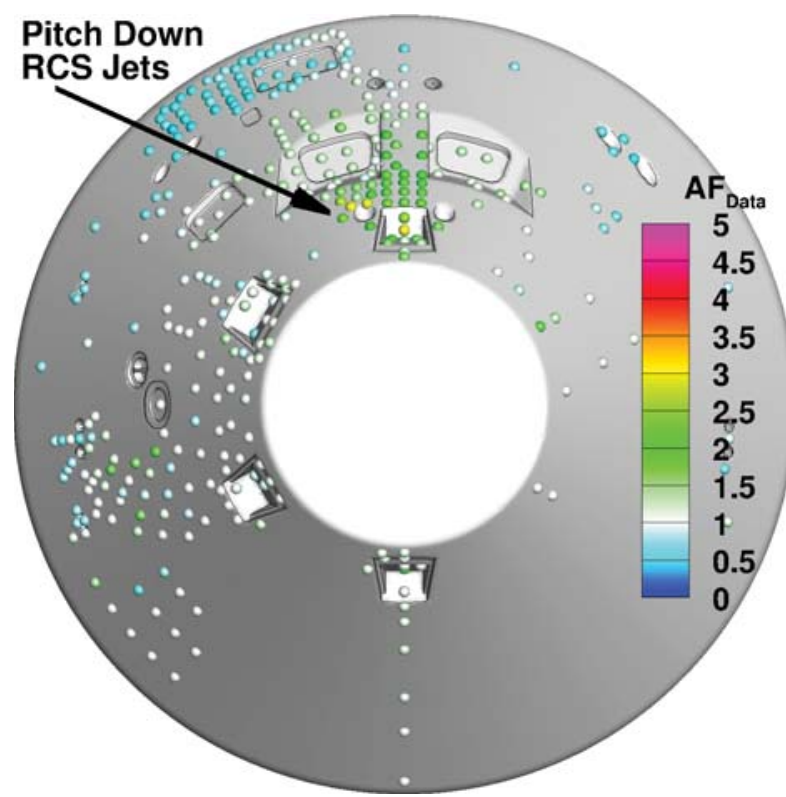

(a) $\mathrm{MR}=0.85 \times 10^{-3}$

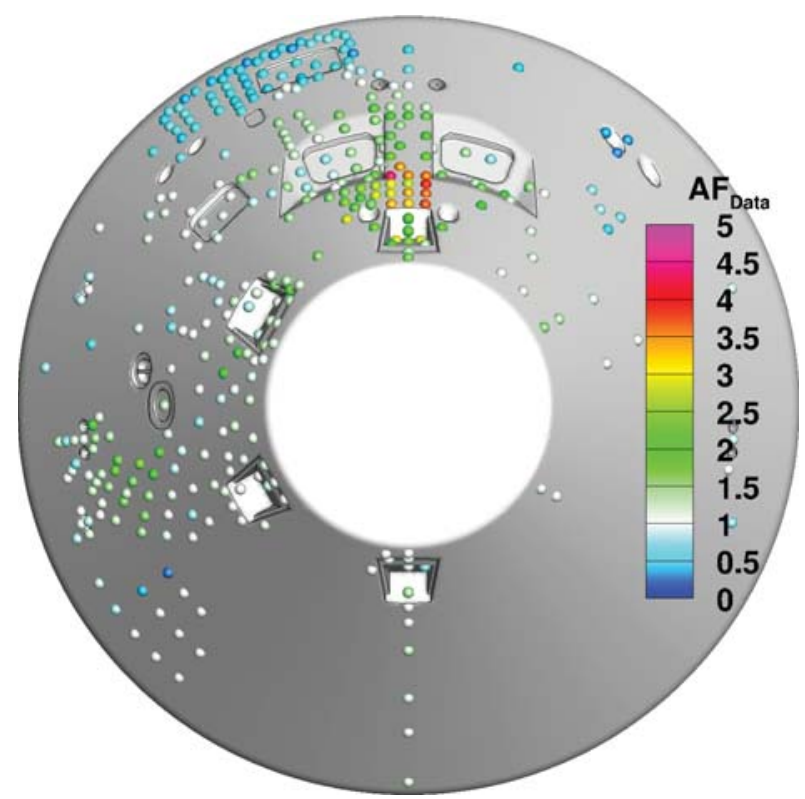

(c) $\mathrm{MR}=1.8 \times 10^{-3}$

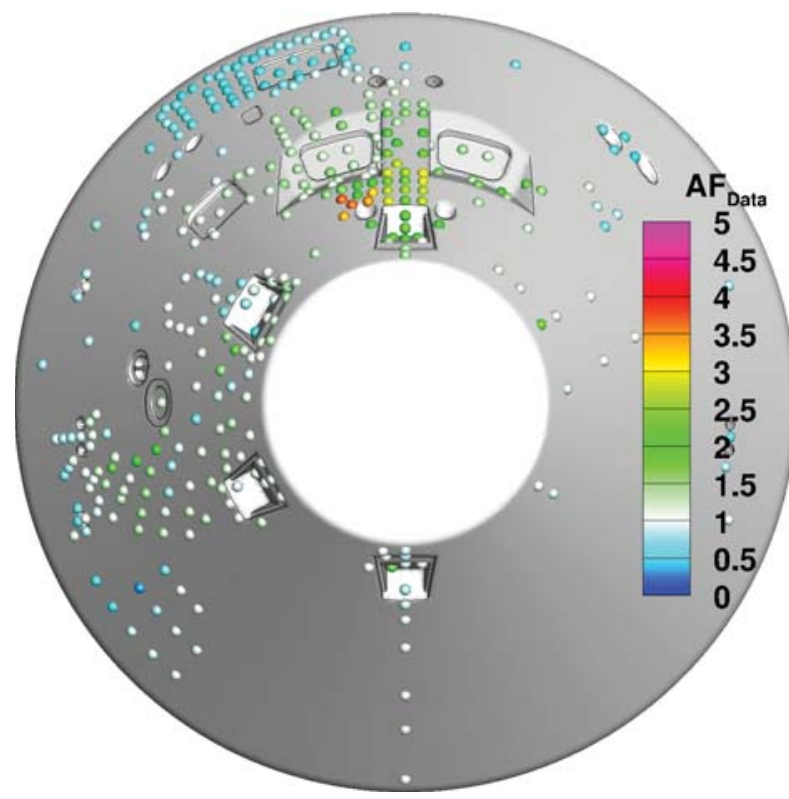

(b) $\mathrm{MR}=1.0 \times 10^{-3}$

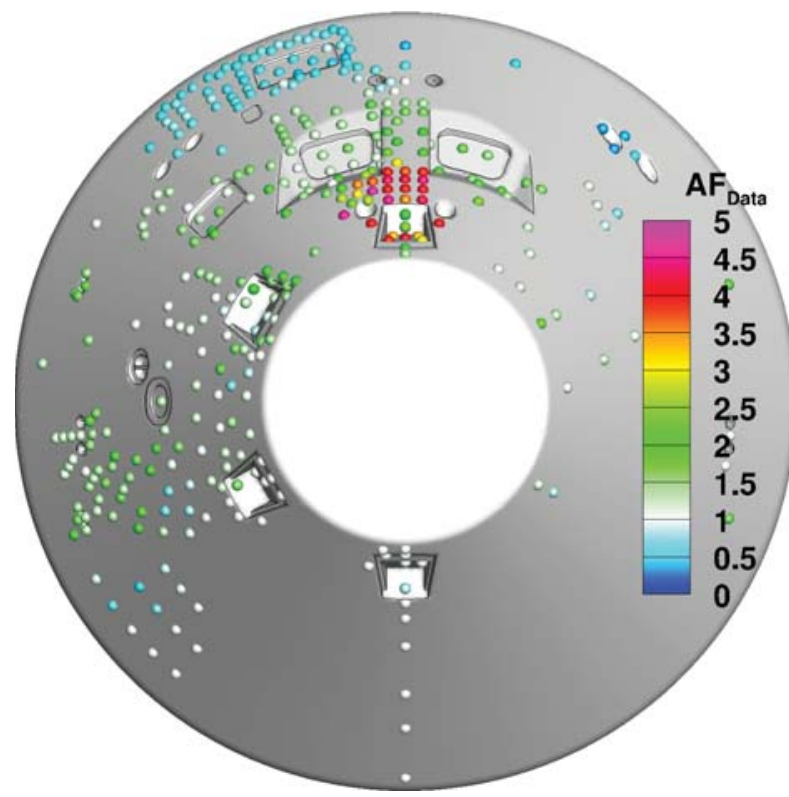

(d) $\mathrm{MR}=2.2 \times 10^{-3}$

Figure 11. Scatter plot of augmentation factor while firing dual pitch down $\mathbf{R C S}$ jets at $\mathbf{M}_{\infty} \approx 8.0, \mathbf{R e}_{\mathbf{D}} \approx$ $\mathbf{2 . 0} \times 10^{6}, \mathrm{AoA}=\mathbf{1 8 . 0}, \mathrm{HR} \approx \mathbf{0 . 5 5}$, while varying momentum ratio

\section{Reynolds Number Trends}

The Reynolds number can vary significantly throughout the potential entry flight space. Within this range it is expected that the attached aft-body will be laminar and turbulent during different portions of the trajectory and the separated wake region will certainly be affected by the variation in $\mathrm{Re}_{\mathrm{D}}$. To insure both a laminar and turbulent attached aft-body were tested, $\mathrm{Re}_{\mathrm{D}}$ was varied from $1.0 \times 10^{6}$ to $20.0 \times 10^{6}$ during the testing. Comparisons with laminar and turbulent CFD at each condition verified that the two lower $\operatorname{Re}_{\mathrm{D}}$ levels $\left(1.0 \times 10^{6}\right.$ and $\left.2.0 \times 10^{6}\right)$ were laminar on the aft-body and the highest $\mathrm{Re}_{\mathrm{D}}$ levels tested with RCS jets firing $\left(11.0 \times 10^{6}\right.$ and $\left.14.0 \times 10^{6}\right)$ were turbulent on the aft-body. Three additional Re $e_{D}$ levels were 


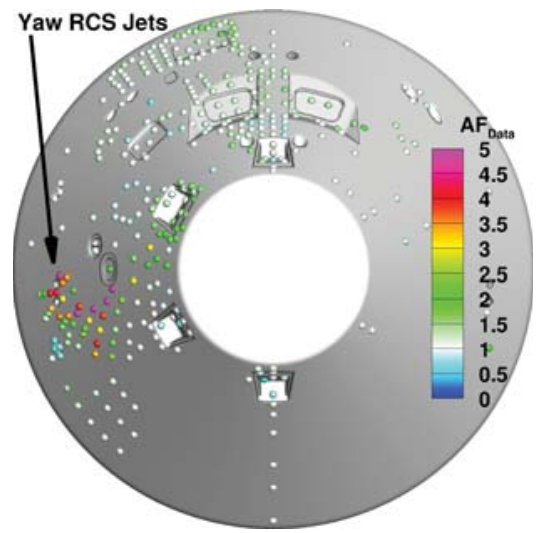

(a) $\mathrm{MR}=0.86 \times 10^{-3}$

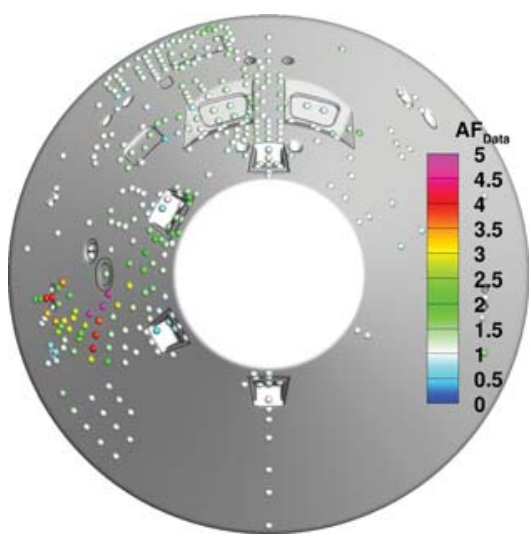

(b) $\mathrm{MR}=0.91 \times 10^{-3}$

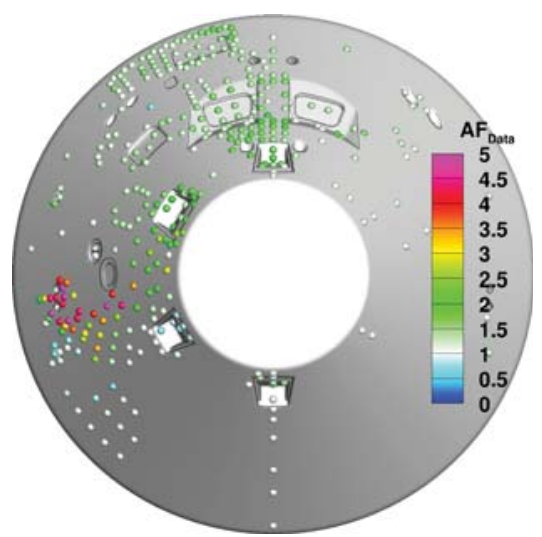

(c) $\mathrm{MR}=1.9 \times 10^{-3}$

Figure 12. Scatter plot of augmentation factor while firing dual yaw RCS jets at $\mathbf{M}_{\infty} \approx \mathbf{8 . 0}, \mathbf{R e}_{\mathrm{D}} \approx \mathbf{2 . 0} \times 10^{6}$, $\mathrm{AoA}=18.0, \mathrm{HR} \approx 0.55$, while varying momentum ratio

also tested between these two extremes at $4.0 \times 10^{6}, 6.0 \times 10^{6}$, and $8.0 \times 10^{6}$. For all of the RCS banks tested for $\mathrm{Re}_{\mathrm{D}}$ dependence (roll, pitch down, and yaw) the lower (laminar) $\mathrm{Re}_{\mathrm{D}}$ level showed qualitatively higher augmentation factors, as shown in Figs. 15, 16, and 17. This was likely due to the plume causing transition of the shear layer and significantly increased mixing across the shear layer when the flow was laminar. At higher $\mathrm{Re}_{\mathrm{D}}$ the $i$ ncrease in the mixing across the shear layer due to the plumes was diminished because the attached flow and likely the shear layer where already transitional or turbulent.

\section{RCS Jet Bank Combinations}

Prior to the 66B-CH test there were no data to develop unique models for combinations of RCS jet banks firing. At that time the models were implemented such that augmentation factor maps associated with each of the jet banks that were being fired would all be active. The final augmentation factor applied at any location would then be taken as the maximum from all of the active maps locally. The 66B-CH data showed that when combinations of jets were fired the resulting heating augmentation could be significantly greater than that seen with any of the associated jets banks firing alone. Figure 18 shows dual roll and single pitch down augmentation factors when firing alone as well as when fired in combination. The peak augmentation factors seen in the region between the large window features and the pitch down nozzles themselves was significantly higher when fired in combination than when fired alone, going from an $\mathrm{AF}_{\text {Data }}=4.2$ when the rolls were fired alone to an $\mathrm{AF}_{\text {Data }}=10.5$ when fired with the pitch down jets. This led to the development of unique augmentation factor maps for jet combinations, which could ultimately drive the peak heat rates seen on portions of the backshell.

\section{E. Discrete Instrumentation}

When the RCS jets were active the resulting heating augmentation could be very localized. This was generally true of all the near jet regions as well as other locations on the vehicle, but was particularly true of the roll RCS near jet region, shown in Fig. 19. The 63-CH test data led to the relatively high clustering of gauges in this region on the 66B-CH test article, which led to the measurement of a peak $\mathrm{AF}_{\text {Data }}=6.5$, shown in Fig. 19a. That peak was only seen at one gauge though and the highest augmentation factor at the surrounding gauges were $\mathrm{AF}_{\text {Data }} \approx 4.0$. This difference led to speculation about both the validity of that one measurement as well as concern that the "true" peak may not have been captured. With that in mind, the instrumentation on the 123-CH part B test article was more tightly clustered around where the peak was measured on the 66B-CH test article, shown in Fig. 19b. The peak augmentation factor seen in this region during 123-CH part $\mathrm{B}$ was $\mathrm{AF}_{\text {Data }}=13.0$. With the added instrumentation several surrounding gauges also measured augmentation factors greater than 4.0 with one measuring $\mathrm{AF}_{\text {Data }} \approx 10.0$, confirming the validity of the measurements in this region. While in general there was greater confidence in the quantitative values from the discrete instrumentation than with the TSP data, the discrete instrumentation were susceptible to 


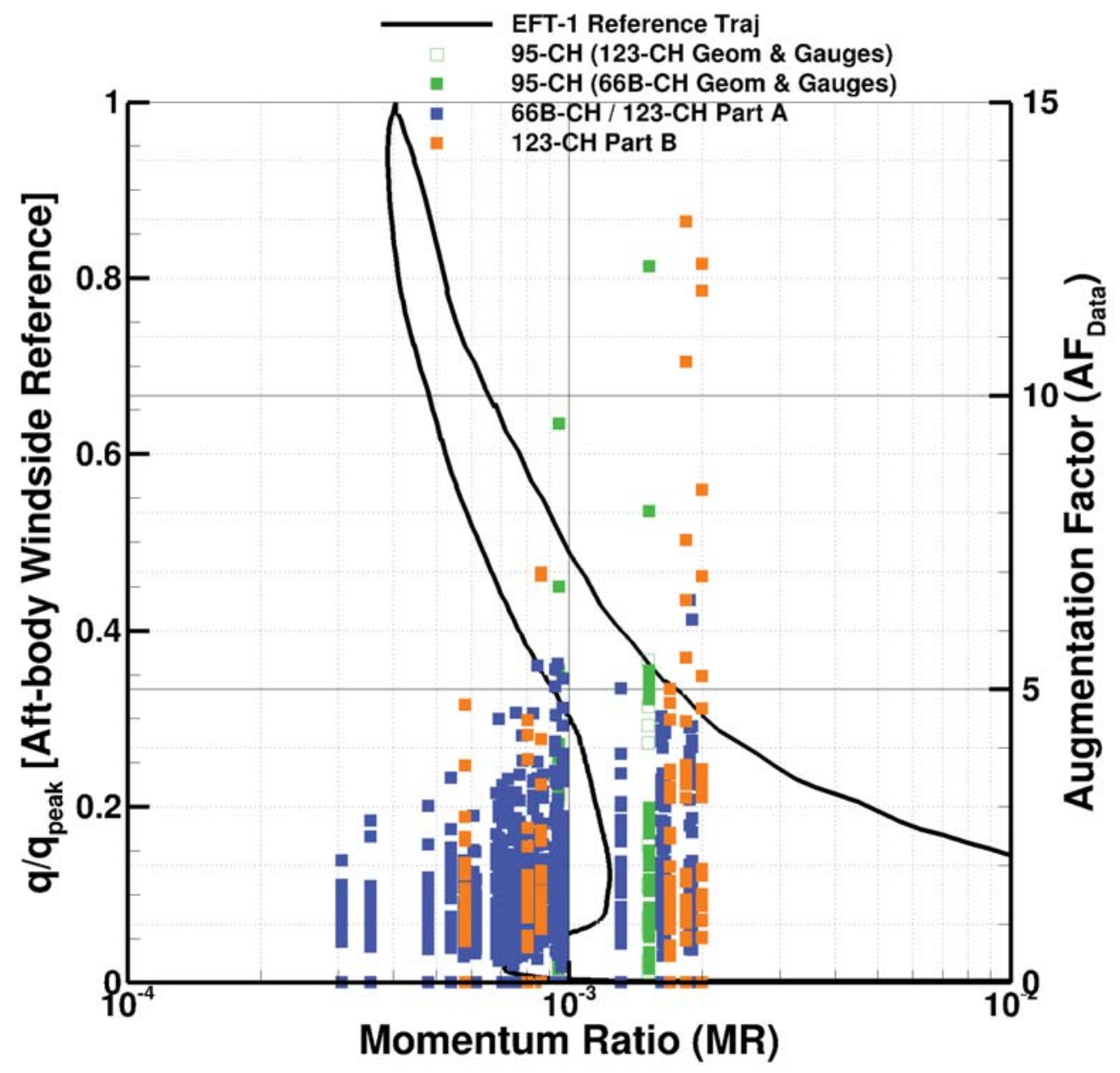

Figure 13. The augmentation factor as a function of momentum ratio in the roll near jet region with dual roll jets firing from the 66B-CH, 95- $\mathrm{CH}$, and $123-\mathrm{CH}$ tests. For reference the normalized heat flux at an aft-body windside location as a function of momentum ratio on an EFT-1 reference trajectory is also shown.

missing highly localized peaks in the heating given that it was difficult to know a priori exactly where the peaks would be located.

\section{F. RCS Influence on Cavity Augmentation}

Prior to the 66B-CH test there were also no data on cavity augmentation in RCS jet influence regions. The two sets of augmentation factor models, RCS jet and cavity augmentation, were developed separately and at that time combined multiplicatively. The $66 \mathrm{~B}-\mathrm{CH}$ data revealed a significant change in the cavity augmentation in the large window features when the roll RCS jets fired, shown in Fig. 20.

The cavity augmentation factors were developed by calculating the ratio of the heat flux level on a gaugeby-gauge basis from a test run when the cavity was exposed to the heat flux level from another run at similar freestream conditions when the cavity was covered.

$$
A F_{\text {cavity }}=\frac{q_{\mathrm{w}, \text { cavity exposed }}}{q_{\mathrm{w}, \text { cavity covered }}}
$$

Cavity augmentation factors were calculated from two runs without RCS jets firing, shown in Fig. 20a, which could be used to check or update cavity models that were used throughout the entry trajectory. Similarly, they could be calculated from two runs, both with RCS jets firing, shown in Fig. 20b, which 


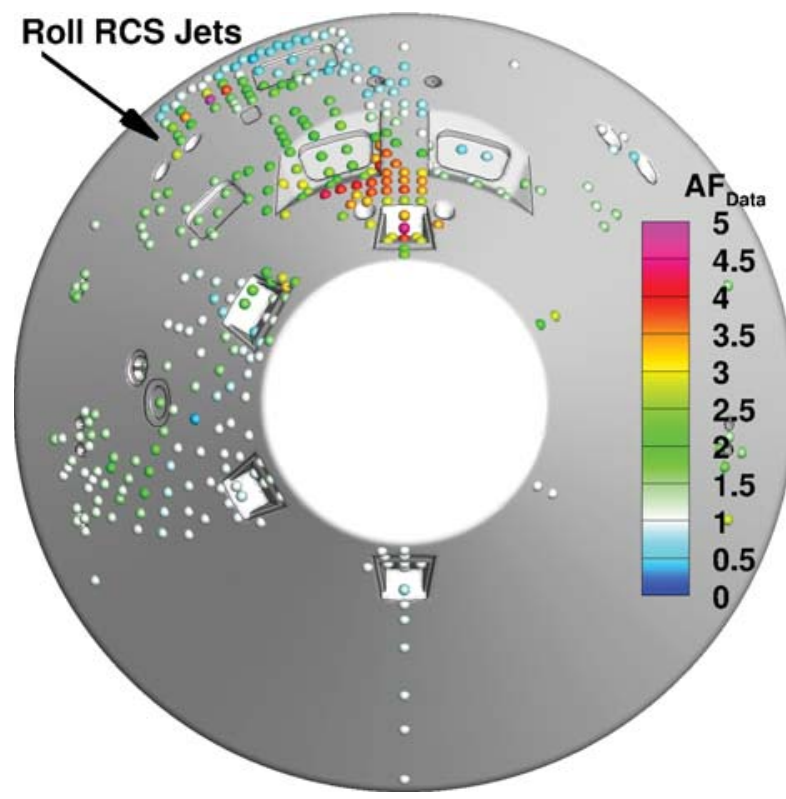

(a) $\mathrm{HR}=0.55$

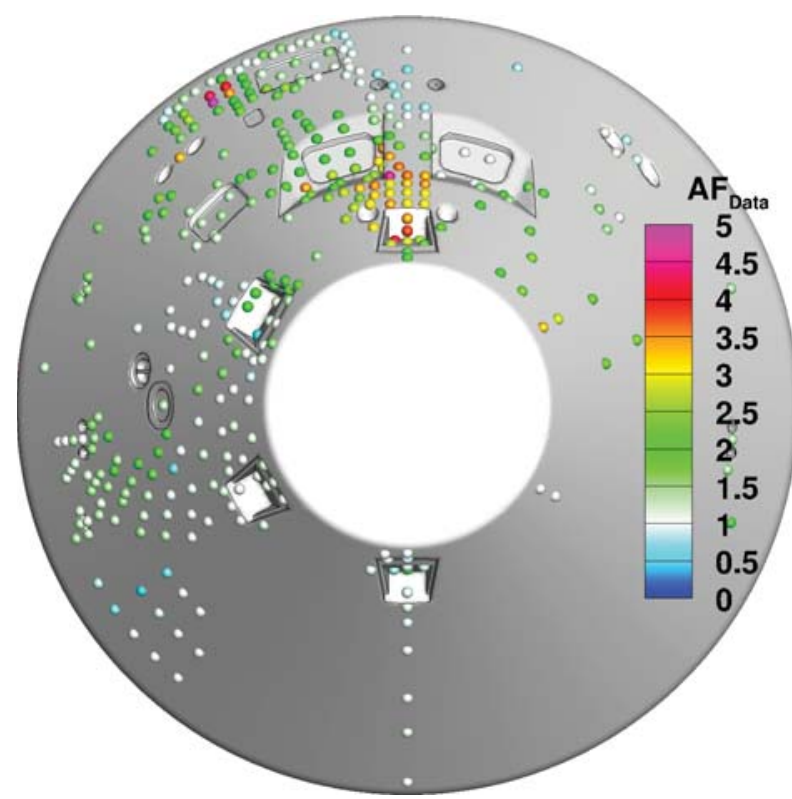

(c) $\mathrm{HR}=0.83$

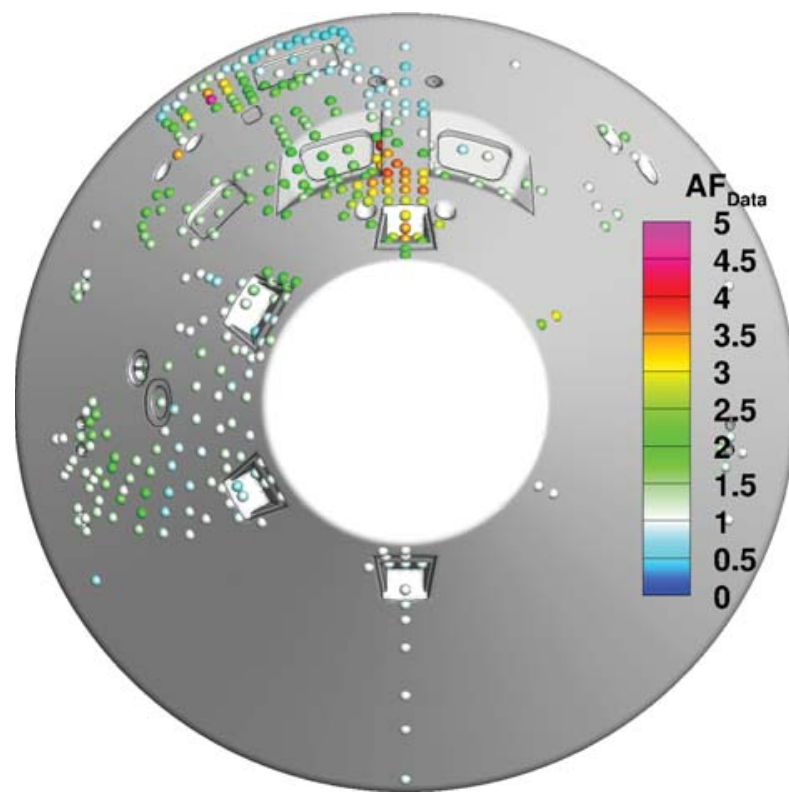

(b) $\mathrm{HR}=0.71$

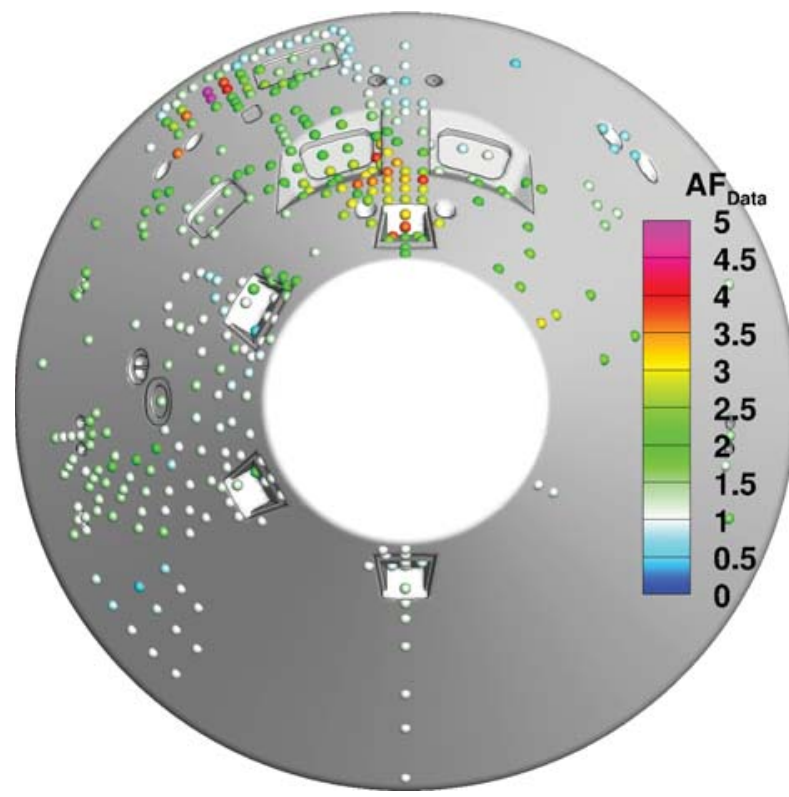

(d) $\mathrm{HR}=0.90$

Figure 14. Scatter plot of augmentation factor while firing dual roll RCS jets at $\mathbf{M}_{\infty} \approx \mathbf{8 . 0}, \mathbf{R e}_{\mathrm{D}} \approx \mathbf{2 . 0} \times 10^{6}$, $\mathrm{AoA}=18.0, \mathrm{MR} \approx 0.9 \times 10^{-3}$, while varying enthalpy ratio

would in principle remove any direct RCS jet augmentation. The differences between the two images in Fig. 20 revealed the influence the roll RCS jets had on the cavity augmentation. The heating augmentation in addition to increasing in general, shifted from the heat shield side inboard corner of the cavity, to the apex side inboard corner and the heat shield side outboard corner. Cavity augmentation is very dependent on the flow-field and in particular the direction the flow is passing over the cavity. The changes to the cavity augmentation when the RCS jets fire were likely due to changes to the wake flow structure around the cavity. This led to a shift in the methodology behind the RCS jet heating augmentation factor model development. Where previously the intended approach was to use the cavity model to augment the predicted heating to account for the real versus smooth OML geometry and the RCS jet model to account for RCS jet heating 


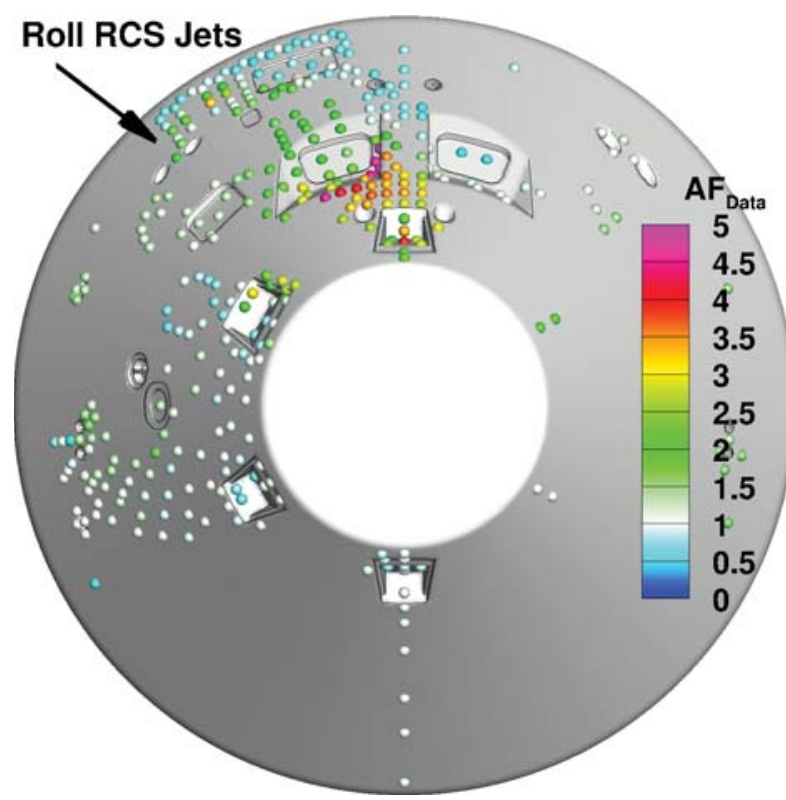

(a) $\operatorname{Re}_{\mathrm{D}} \approx 2.0 \times 10^{6}$

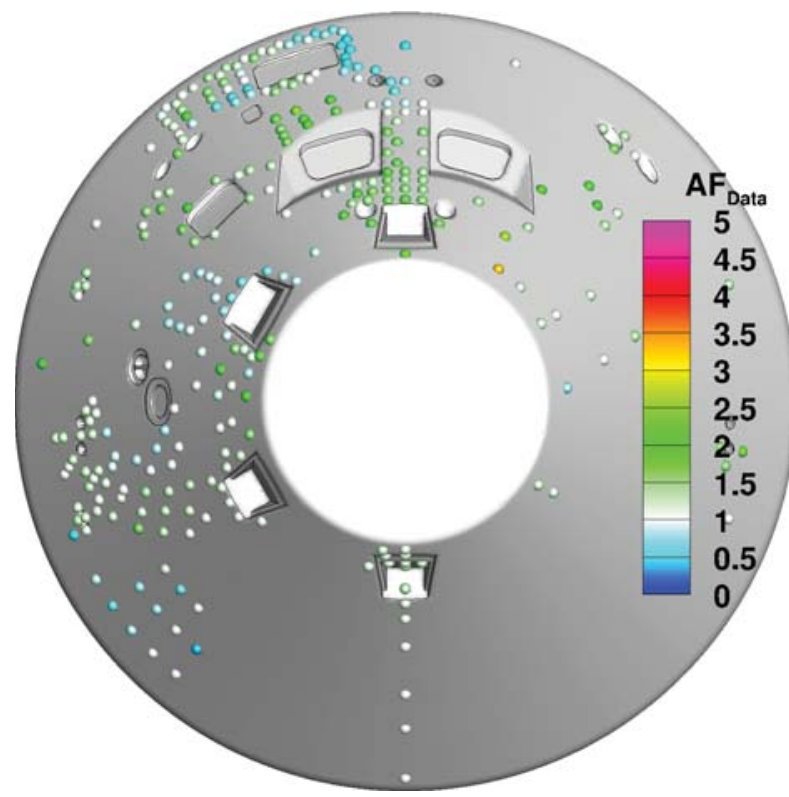

(b) $\operatorname{Re}_{\mathrm{D}} \approx 14.0 \times 10^{6}$

Figure 15. Scatter plot of augmentation factor while firing dual roll RCS jets at $\mathrm{M}_{\infty} \approx \mathbf{8 . 0}, \mathrm{AoA}=18.0$, MR $\approx 0.65 \times 10^{-3}, \mathrm{HR} \approx 0.55$, while varying freestream $\mathrm{Re}_{\mathrm{D}}$

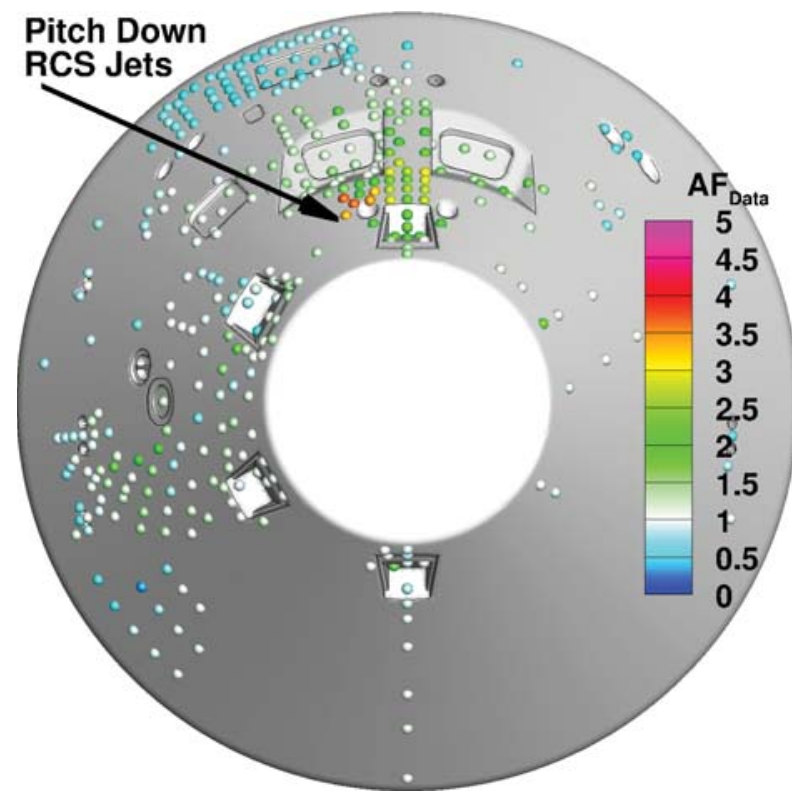

(a) $\operatorname{Re}_{\mathrm{D}} \approx 2.0 \times 10^{6}$

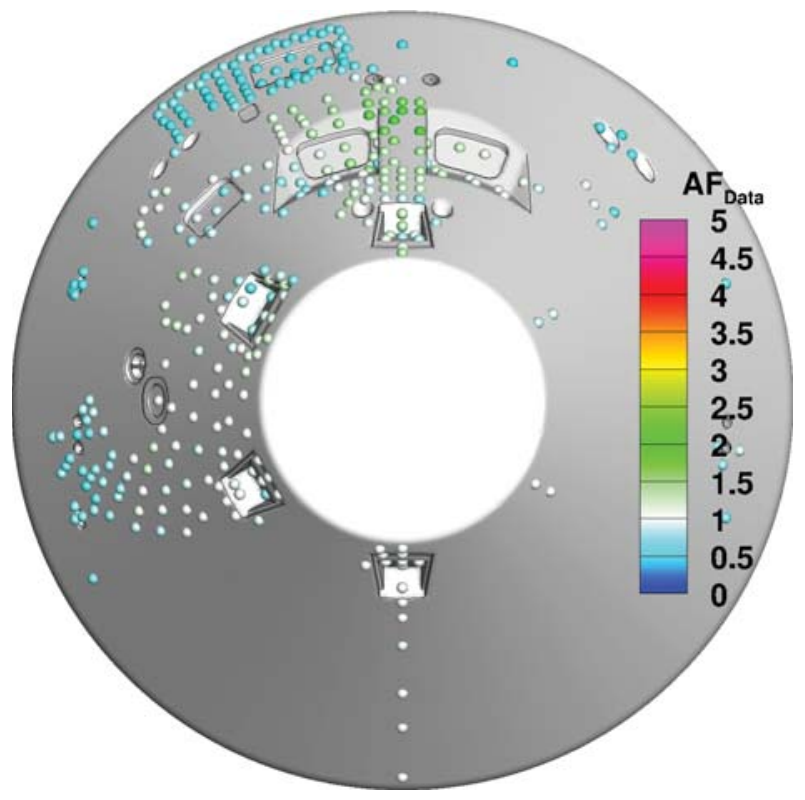

(b) $\operatorname{Re}_{\mathrm{D}} \approx 11.0 \times 10^{6}$

Figure 16. Scatter plot of augmentation factor while firing dual pitch down $\mathrm{RCS}$ jets at $\mathbf{M}_{\infty} \approx \mathbf{8 . 0}, \mathbf{A o A}=18.0$, $\mathrm{MR} \approx 0.8 \times 10^{-3}, \mathrm{HR} \approx 0.55$, while varying freestream $\mathbf{R e}_{\mathrm{D}}$

augmentation to the real geometry:

$$
A F_{\text {Final }}=A F_{\text {cavity }} \times A F_{\mathrm{RCS}}=\frac{q_{\mathrm{w}, \text { no RCS, cavity exposed }}}{q_{\mathrm{w}, \text { no RCS, cavity covered }}} \times \frac{q_{\mathrm{w}, \text { RCS, cavity exposed }}}{q_{\mathrm{w}, \text { no- } \mathrm{RCS}, \text { cavity exposed }}}
$$

The new methodology used the RCS jet heating augmentation factor model to accommodate both the 


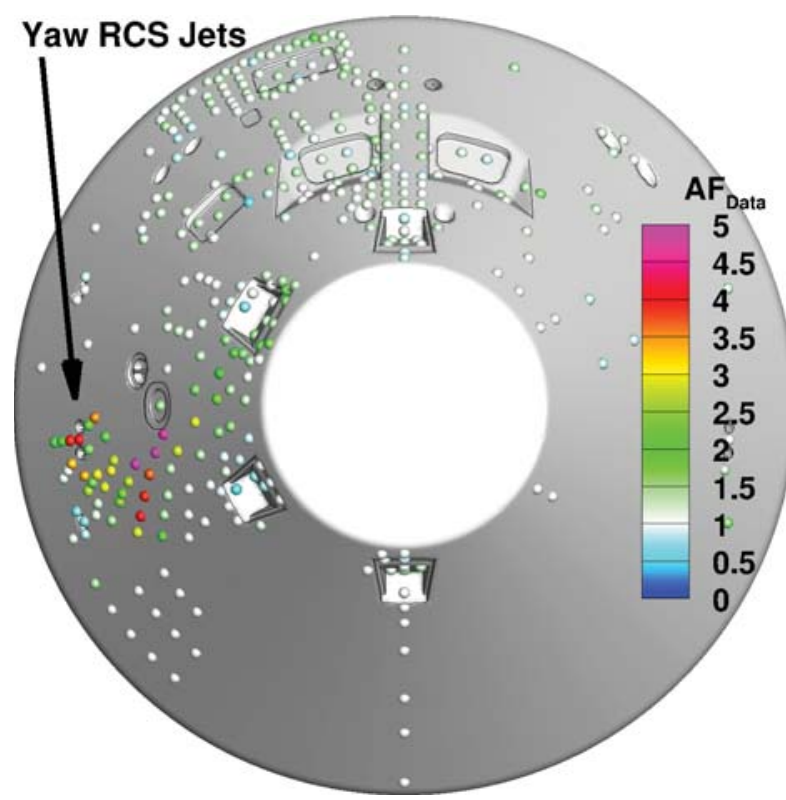

(a) $\operatorname{Re}_{\mathrm{D}} \approx 2.0 \times 10^{6}$

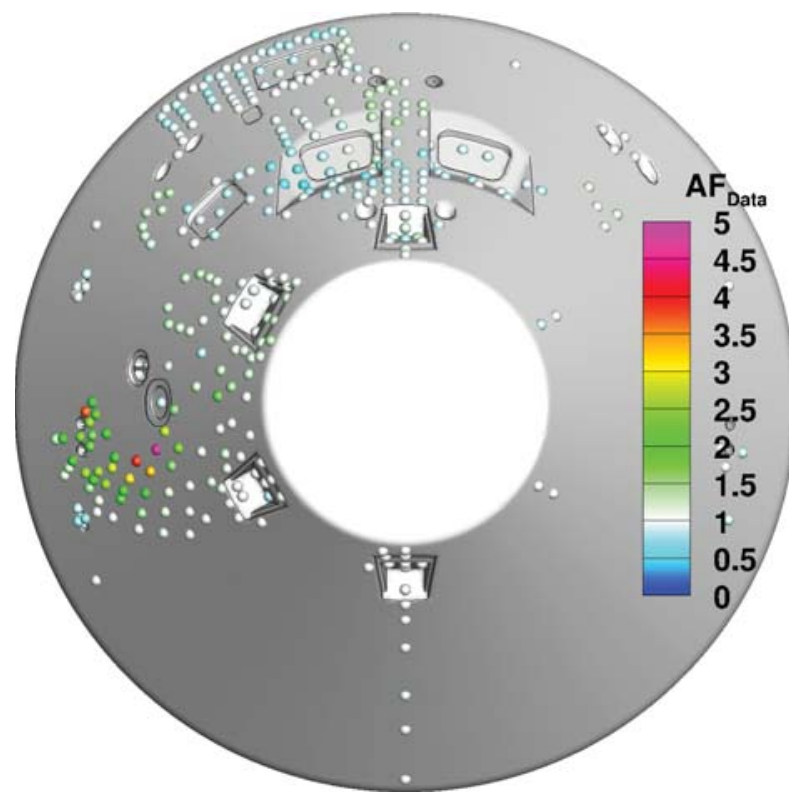

(b) $\operatorname{Re}_{\mathrm{D}} \approx 11.0 \times 10^{6}$

Figure 17. Scatter plot of augmentation factor while firing dual yaw RCS jets at $\mathrm{M}_{\infty} \approx \mathbf{8 . 0}, \mathrm{AoA}=18.0, \mathrm{MR}$ $\approx 0.8 \times 10^{-3}, \mathrm{HR} \approx \mathbf{0 . 5 5}$, while varying freestream $\mathrm{Re}_{\mathrm{D}}$

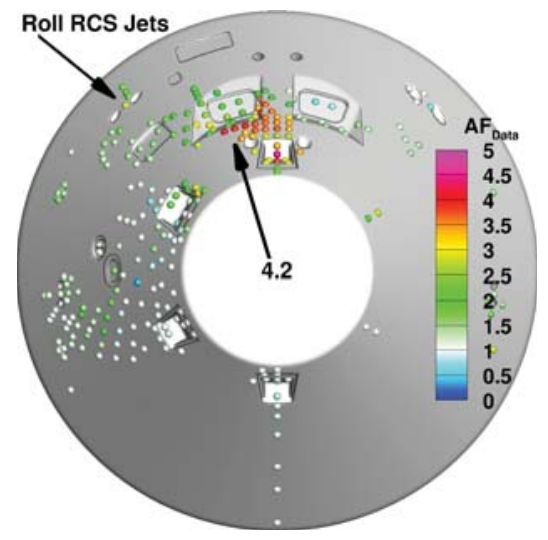

(a) Dual Roll

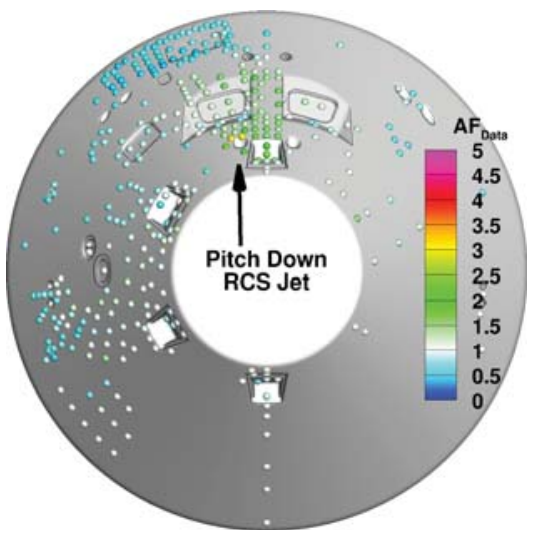

(b) Single Pitch Down

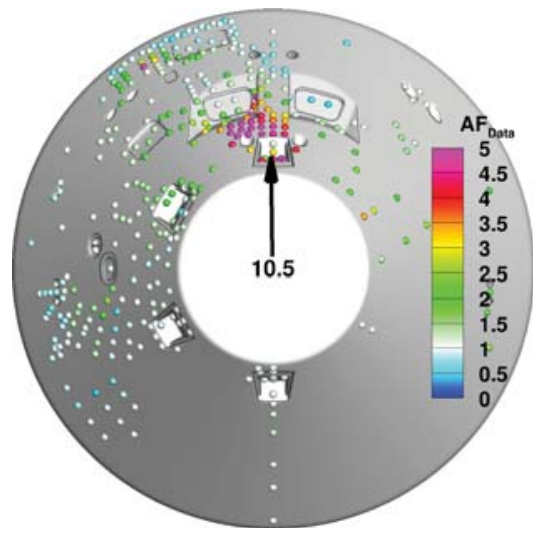

(c) Combination Dual Roll with Single Pitch Down

Figure 18. Scatter plots of $A_{F_{R C S}}$, illustrating the impact of RCS jet combinations on the $A_{\text {RCS }}$ compared to the same jets firing individually.

change in geometry and the RCS jet influence when the RCS were active:

$$
A F_{\text {Final }}=A F_{\mathrm{RCS}}=\frac{q_{\mathrm{w}, \mathrm{RCS}, \text { cavity exposed }}}{q_{\mathrm{w}, \text { no-RCS, } \text {, cavity covered }}}
$$




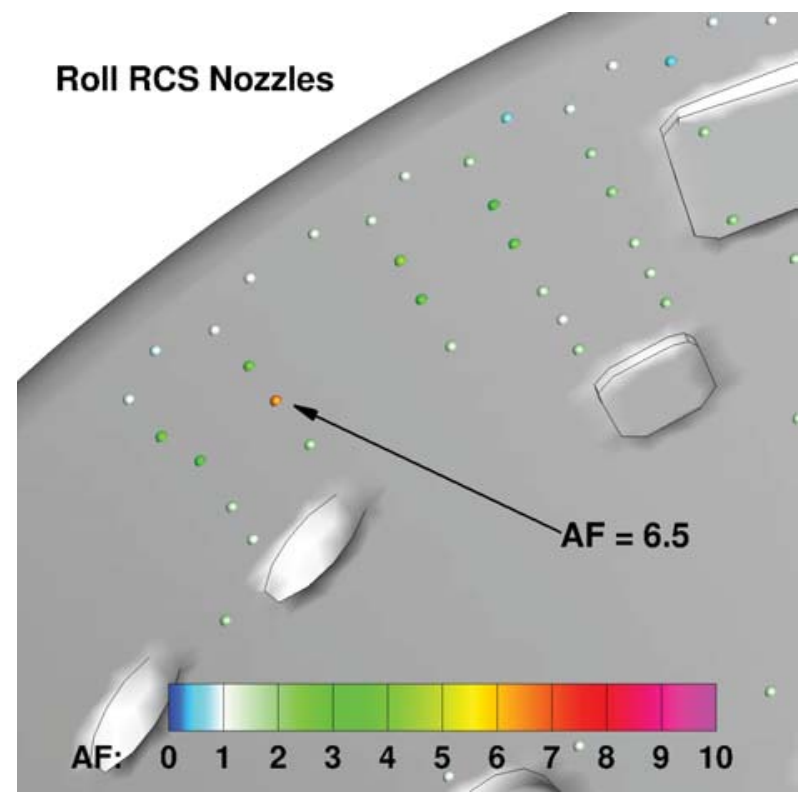

(a) 66B-CH Peak

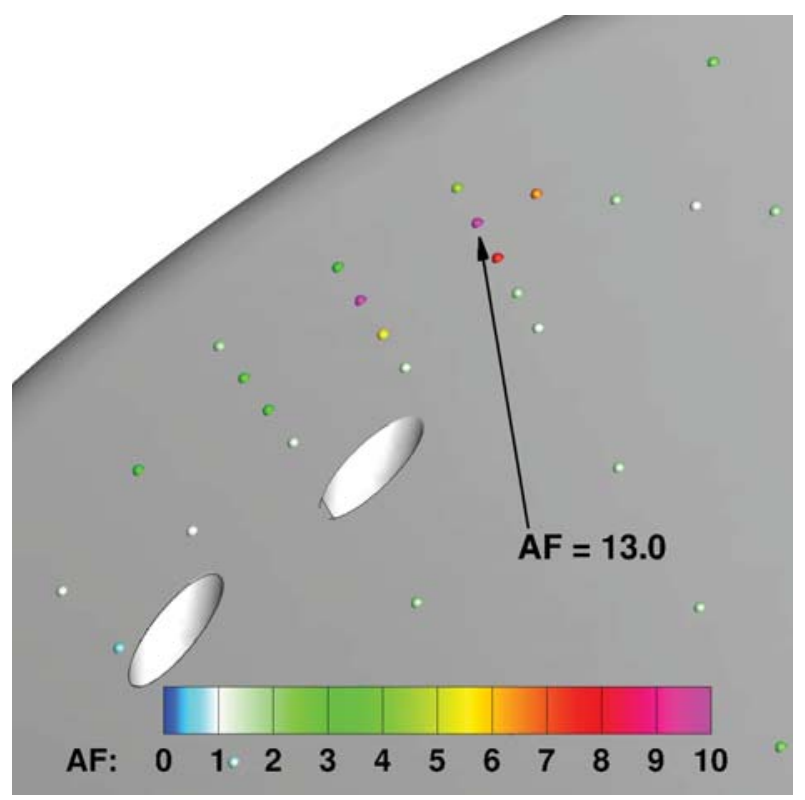

(b) 123-CH Part B Peak

Figure 19. Scatter plot of roll near jet region augmentation factors with dual roll firing. The maximum heating augmentation factors in this region were very localized.

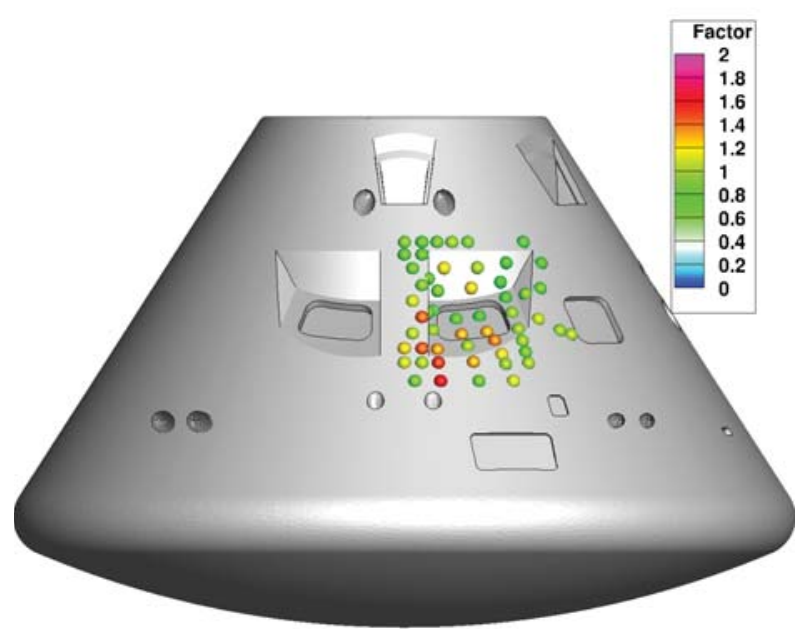

(a) No RCS jets firing

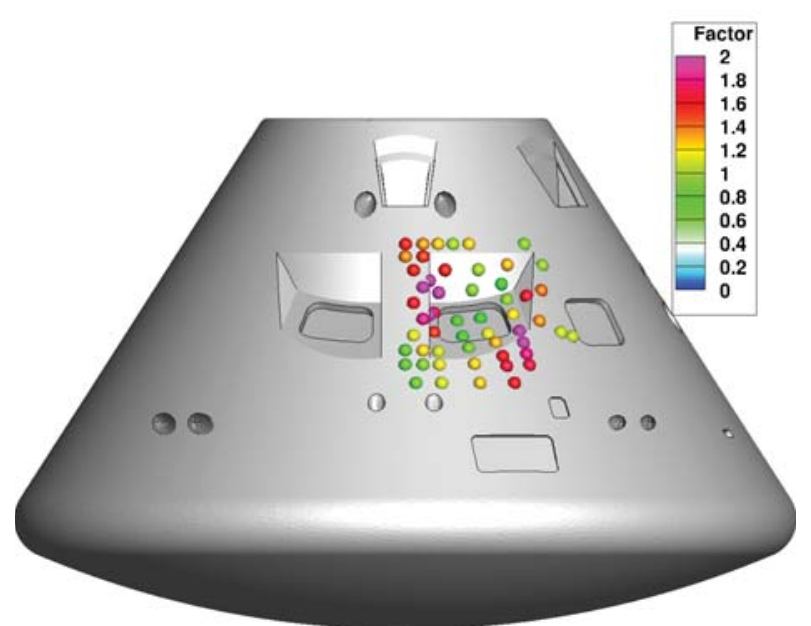

(b) Dual roll RCS jets firing

Figure 20. Scatter plot of cavity augmentation factor $\left(\mathbf{A F}_{\text {cavity }}\right)$.

\section{Model Development}

The sensitivity of RCS environments to configuration, geometry and orientation made it difficult to take advantage of any existing flight data from Apollo or other missions in the development of the Orion MPCV RCS jet interaction (JI) models. Additionally, while the Apollo CM had a number of backshell thermocouples, the recent wind tunnel tests showed there could be very localized peaks in the heating when 
the RCS jets fired and those peaks were likely not captured by the Apollo flight instrumentation. The dearth of applicable flight-data, coupled with the limited success of CFD in this complicated flow-field meant the MPCV RCS jet heating augmentation factor models would need to be developed solely from the available wind tunnel test data.

The wind tunnel data demonstrated that the RCS jet heating augmentation factors had significant spatial variation and were strongly dependent on freestream condition and jet condition. As discussed already, it was decided not to utilize the variation seen within the trajectory space due to a number of uncertainties. On the other hand, it was important to utilize the spatial variation seen in the wind tunnel data, given that portions of the separated wake region saw no RCS jet influence with some or all of the RCS jet banks. The spacial variation was accommodated by developing a series of surface maps of augmentation factor as a function of location.

\section{A. Augmentation Factor Calculation}

As discussed previously, the $66 \mathrm{~B}-\mathrm{CH}$ and $123-\mathrm{CH}$ test programs provided the quantitative data used to develop the RCS jet heating augmentation factor models. The data from these tests were initially evaluated using a data-to-data augmentation factor $\left(\mathrm{AF}_{\text {Data }}\right)$ calculation. In this method, the measured heating when the RCS jets were fired was divided by the measured heating when the RCS jets were not fired (Eqn. (3)) at every gauge location. The smooth OML heating level in the flight database was anchored to turbulent CFD solutions. In order to remain consistent with how the $\mathrm{AF}_{\mathrm{RCS}}$ were to be used in flight, the data were normalized by turbulent smooth OML CFD heating predictions at wind tunnel conditions for the development of the RCS jet augmentation factor models (Eqn. (7)).

$$
A F_{\mathrm{CFD}}, \text { Turb }=\frac{q_{w, \text { RCS-on,data }}}{q_{w, \text { RCS-off, Smooth OML CFD }} \text { turb }}
$$

The augmentation factors $\left(\mathrm{AF}_{\text {Data }}\right)$ seen at lower (laminar) $\mathrm{Re}_{\mathrm{D}}$ levels were generally higher than those seen at higher (turbulent) $\mathrm{Re}_{\mathrm{D}}$ levels, as discussed previously in Section IV.C. At higher (turbulent) $\operatorname{Re}_{\mathrm{D}}$ levels, the no RCS firing data and the corresponding turbulent smooth OML CFD became more consistent. This meant that as the conservatism in the smooth OML prediction was reduced at turbulent $\mathrm{Re}_{\mathrm{D}}$ levels, the potential conservatism in the RCS model from applying a bounding augmentation factor $\left(\mathrm{AF} \mathrm{CFD}_{\text {, Turb }}\right.$ ) that remains constant in flight space could be greater.

\section{B. Compiled Maximum Augmentation Approach}

The wind tunnel test data were examined to identify trends with potential similarity parameters, like those seen with the jet-to-freestream momentum ratio. But with no means to substantiate these trends at flight conditions, they could not be utilized in the model. Therefore a bounding approach was adopted and became referred to as the Compiled Max Augmentation Factor approach. For a given RCS selection (like dual right roll jets), all of the relevant wind tunnel runs were evaluated to determine the maximum augmentation factor at each gauge. The $123-\mathrm{CH}$ part B and the $66 \mathrm{~B}-\mathrm{CH} / 123-\mathrm{CH}$ part A data sets were calculated independently as they were on different geometries and had different gauge layouts. The Compiled Max Augmentation Factor plots for the individual jet banks (single and dual firings calculated separately) and for each test article are shown in Figs. 21, 22, 23, 24 and 25. These plots show the maximum measured augmentation factor $\left(\mathrm{AF}_{\mathrm{CFD}}\right.$, Turb) at each gauge for a given RCS jet, while varying freestream and jet-tofreestream parameters. The pitch up and yaw jet banks were not included on the 123-CH part B test article and only have Compiled Max Augmentation Factor results from the 66B-CH / 123-CH part A test series. While augmentation factor trends were not utilized in the developed models, using the trends to extrapolate from the test space to the regions of the flight space that could not be tested helped build confidence that the models would indeed bound the flight environments.

The Compiled Max Augmentation Factor results for the roll RCS jets are shown in Fig. 21. The previous aft-body cone angle results (Fig. 21 (a) and (c)) on the 66B-CH / 123-CH part A geometry show lower heating augmentation values than the 123-CH part B results (Fig. 21 (b) and (d)) on the current aft-body cone angle. As discussed previously this is believed to be partially due to increased resolution of the discrete instrumentation in critical locations and possibly also due to the angle change. The single roll firings show a peak near jet heating augmentation $\left(\mathrm{AF}_{\mathrm{CFD}}\right.$, Turb $)$ of 4.7 , considerably less than the dual roll firings, which show a peak near jet $\mathrm{AF}_{\mathrm{CFD}}$, turb of 8.2 . 


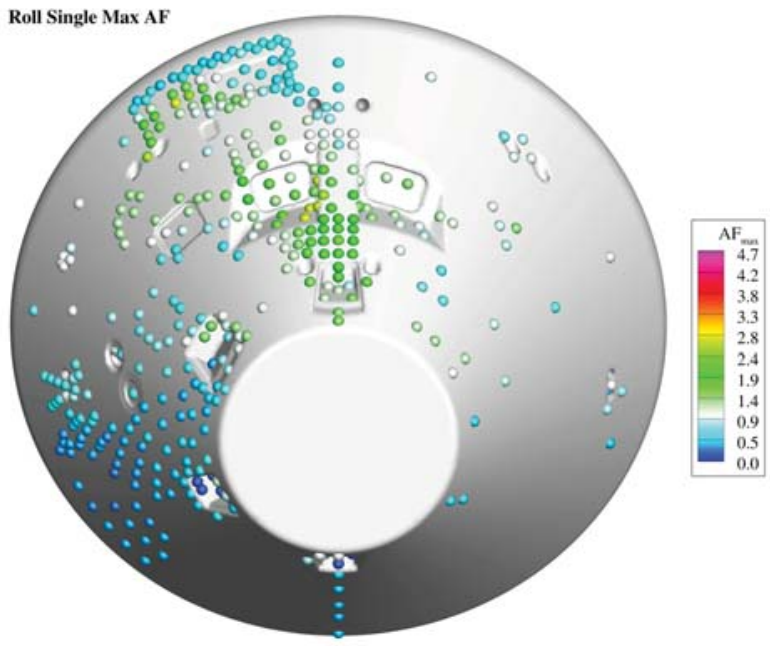

(a) Single Roll Right from 66B-CH / 123-CH Part A

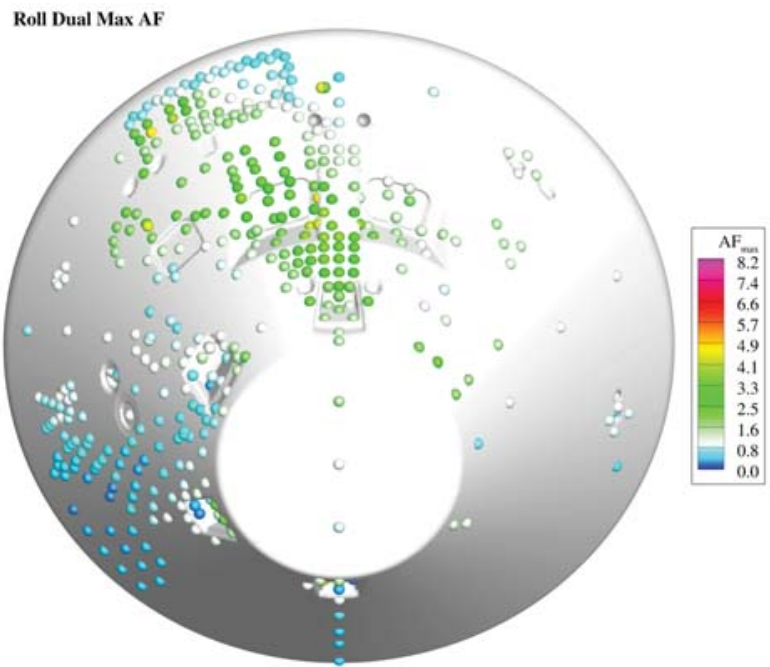

(c) Dual Roll Right from 66B-CH / 123-CH Part A

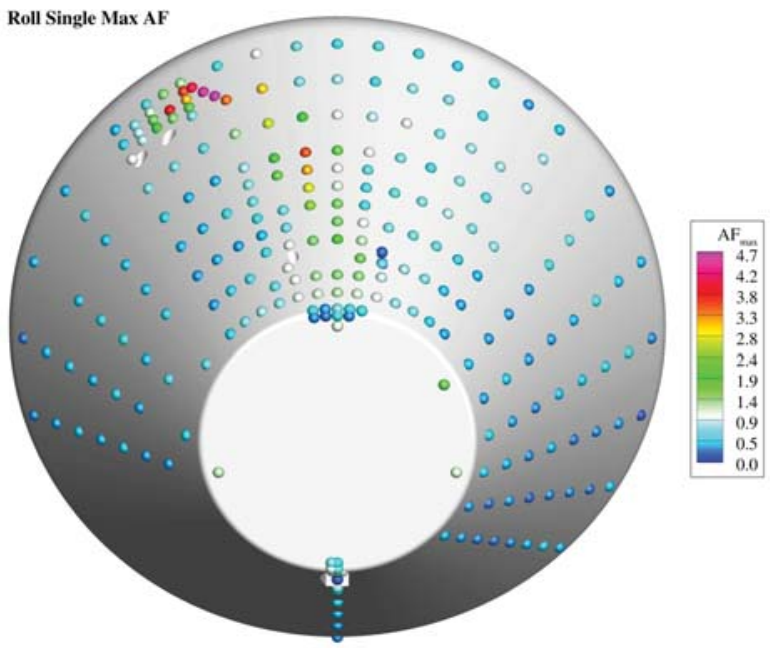

(b) Single Roll Right from 123-CH Part B

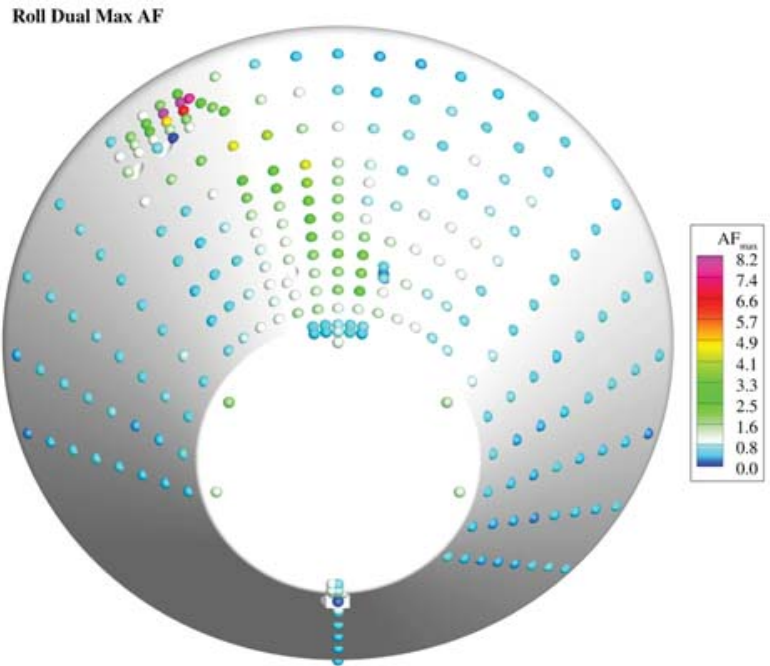

(d) Dual Roll Right from 123-CH Part B

Figure 21. Roll RCS jet Compiled Max Augmentation Factor (AF CFD, Turb).

\section{Map Areas}

With the augmentation factor formulation selected coupled with the Compiled Max Augmentation Factor approach, the areas of influence for each RCS jet bank could be identified and subsequently divided into regions. It was decided to limited the developed augmentation factor maps to only increase the heating. While the data showed some regions saw relief in the heating when the RCS jets were fired, the models did not take advantage of that relief. This was again due to uncertainties in flight traceability and sting effects. The final map areas, determined using some engineering judgement, were intentionally developed to be slightly conservative with respect to the extent of the RCS jet influence region seen in the data.

The map areas were then divided into regions, each with a different magnitude of augmentation factor driven by the data applicable to that region. In general the intention was to use the regions to accommodate the heating associated with specific flow phenomena, like the very localized high heating augmentation seen near the roll RCS jet exit, while allowing the more extensive heating augmentation resulting from increased mixing across the shear layer to be covered by the remaining region. As a result, the higher heating region 


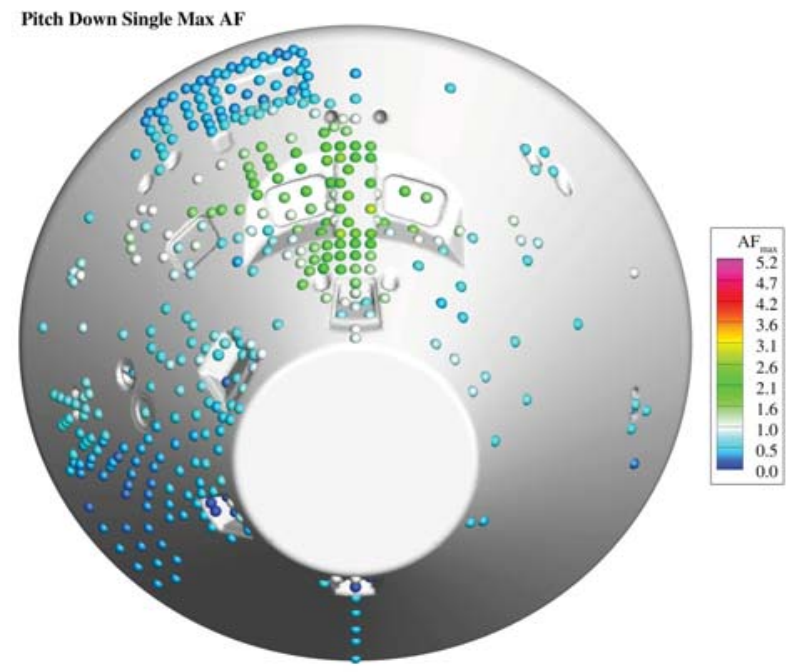

(a) Single Pitch Down from 66B-CH / 123-CH Part A

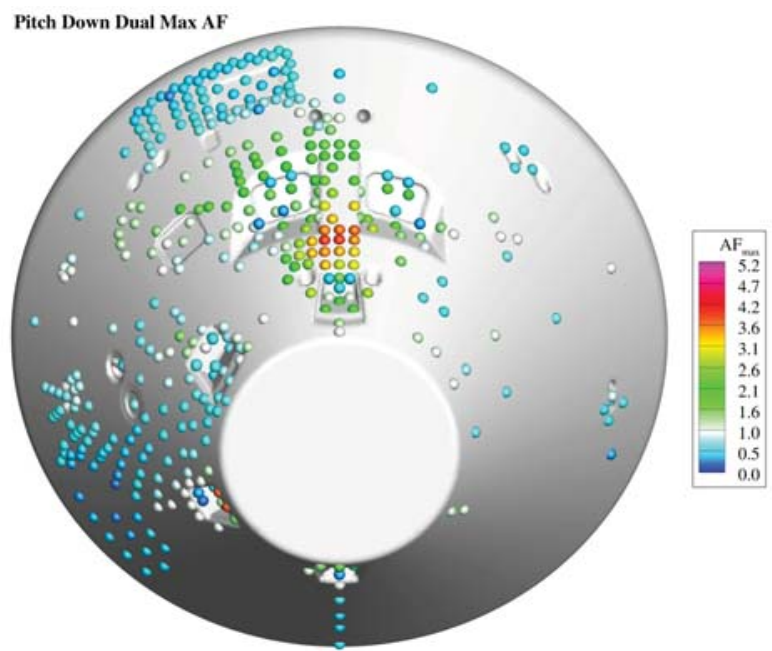

(c) Dual Pitch Down from 66B-CH / 123-CH Part A

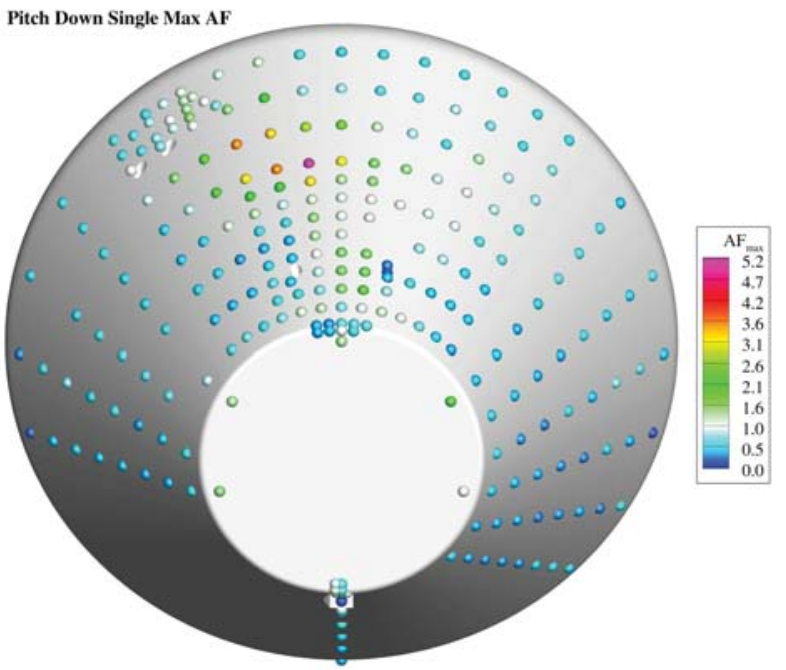

(b) Single Pitch Down from 123-CH Part B

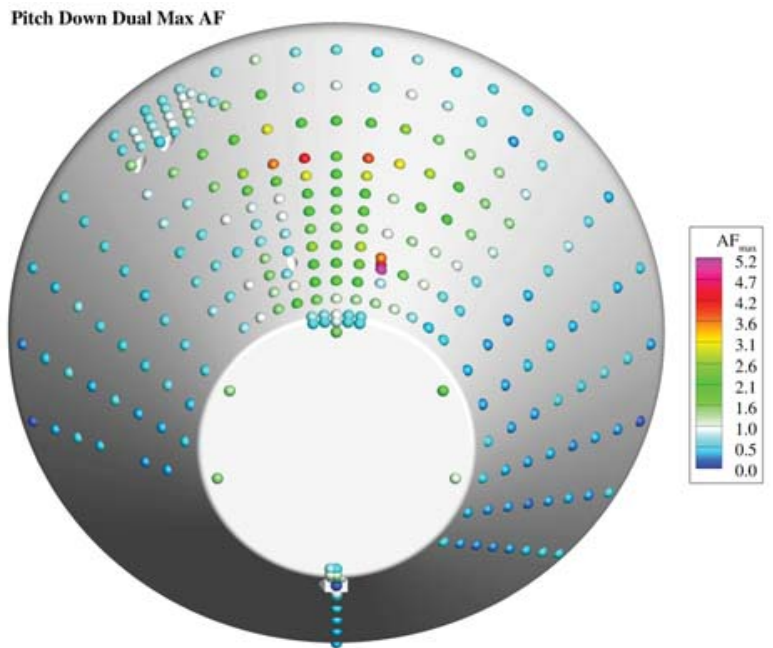

(d) Dual Pitch Down from 123-CH Part B

Figure 22. Pitch Down RCS Jet Compiled Max Augmentation Factor (AF $\left.\mathbf{A F D}_{\text {, Turb }}\right)$.

that abutted the RCS jet itself became known as the near jet region. That said, in the case of the pitch jets, the localized high augmentation was not as pronounced. The pitch down jets were deep in the subsonic separated wake so there was no bow shock near the surface to drive the very localized higher heating. This led to regions of higher heating augmentation that dominated the influence area, and the smaller region of lower heating augmentation really being developed more to avoid being overly conservative. The localized heating augmentation near the yaw jet was also difficult to isolate due to the proximity of the yaw jets to the separation line. The augmentation factors seen along the separation zone were roughly equal to those measured very near the jets themselves, which led to fairly large high heating augmentation factor regions for the yaws as well. Additionally, if there was the potential for the near jet interaction to sit on multiple TPS materials the near jet region was made to include each of the materials. 


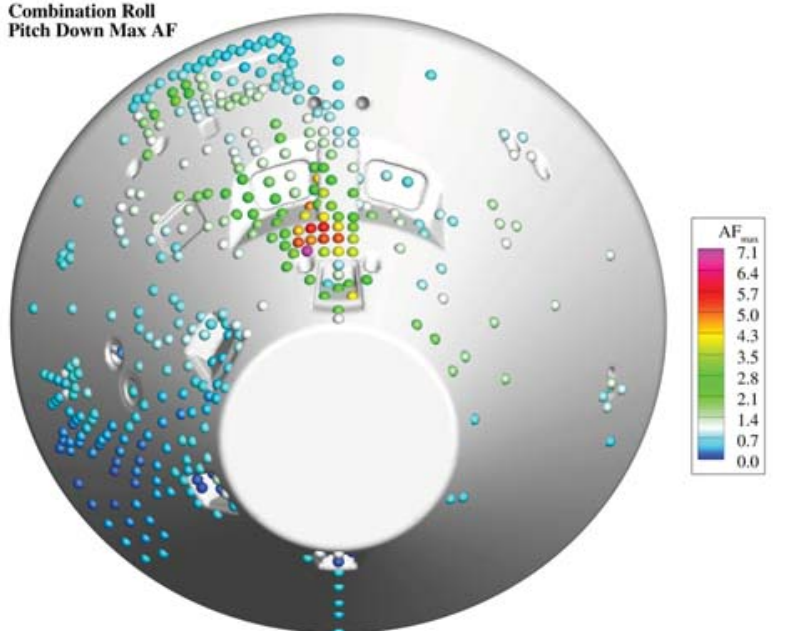

(a) Combination Roll Right and Pitch Down from 66B-CH / 123-CH Part A

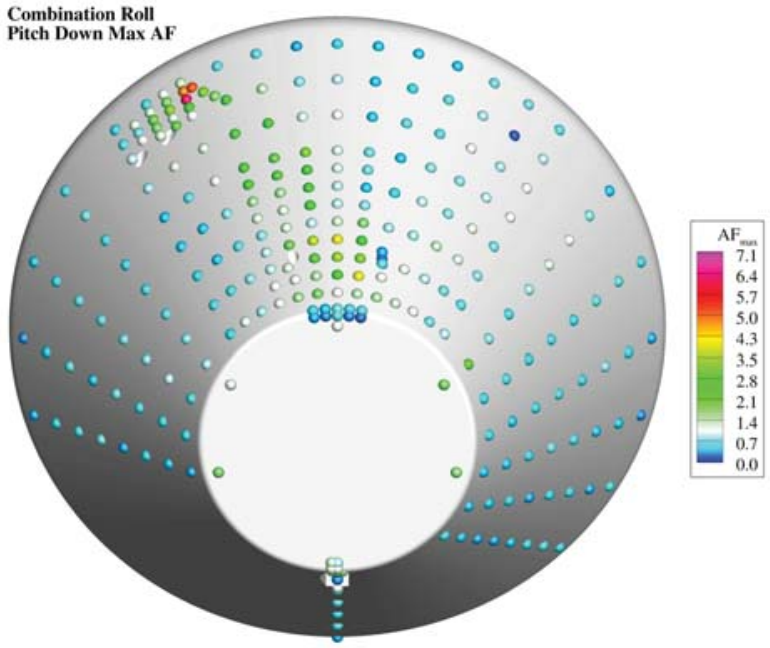

(b) Combination Roll Right and Pitch Down from 123-CH Part B

Figure 23. Combination RCS Jet Compiled Max Augmentation Factor (AF $\mathbf{C F D}_{\mathbf{C F}}$, Turb).

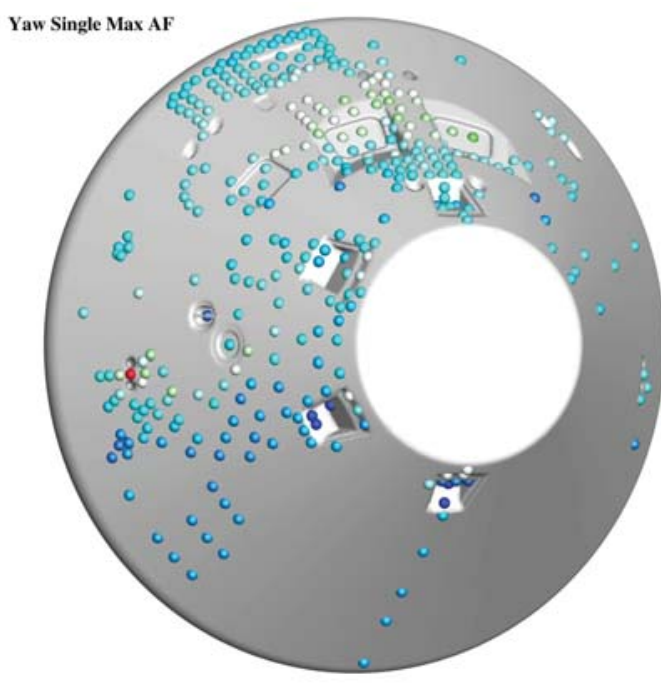

(a) Single Yaw from 66B-CH

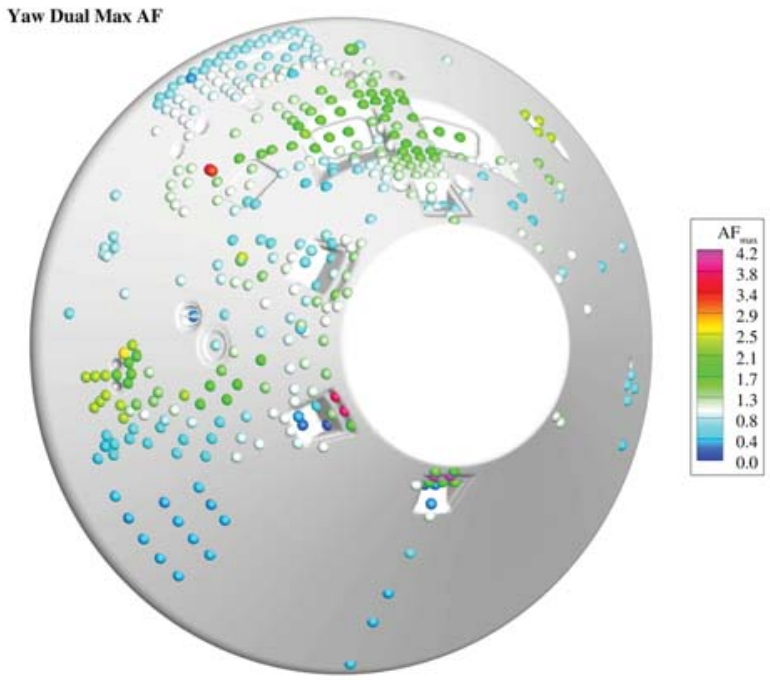

(b) Dual Yaw from 66B-CH

Figure 24. Yaw RCS Jet Compiled Max Augmentation Factor (AF CFD, Turb).

\section{Factor Magnitudes}

Once the map areas for each RCS bank were established and the regions within those areas identified, the augmentation factor magnitude assigned to each region could be determined. The Compiled Maximum Augmentation Factor plots were overlaid with the map areas and the highest $\mathrm{AF}_{\mathrm{CFD}}$, turb in each region was selected. While the team recognized the conservatism in this approach, they also recognized the sensitivity of the RCS interactions to a multitude of parameters, and consequently the possibility that the interactions and associated heating would move appreciably in flight as compared to what were seen in the data. Figures 26, 27, 28, 29, and 30 show the final augmentation factors and areas for each RCS jet bank overlaid on the individual Compiled Maximum Augmentation Factor plots. Table 3 provides a summary of the heating 


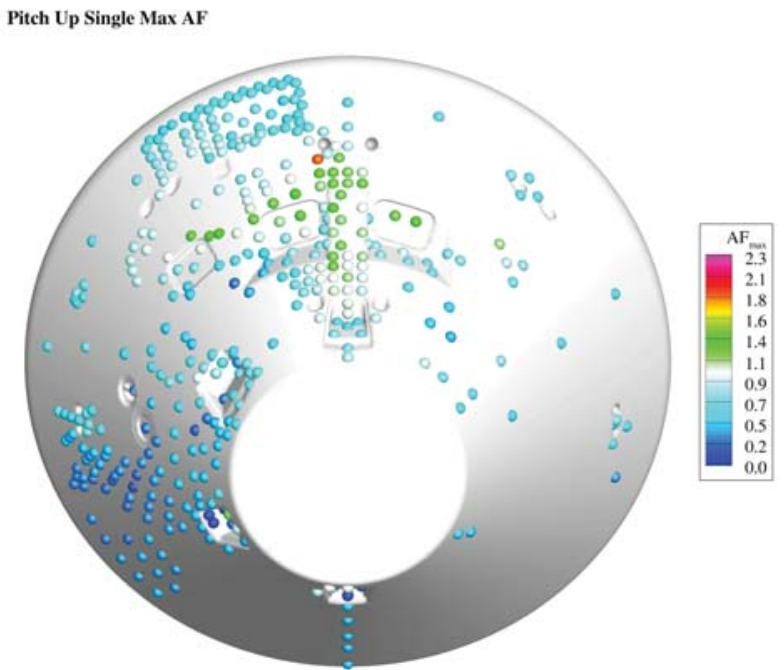

(a) Single Pitch Up from 66B-CH

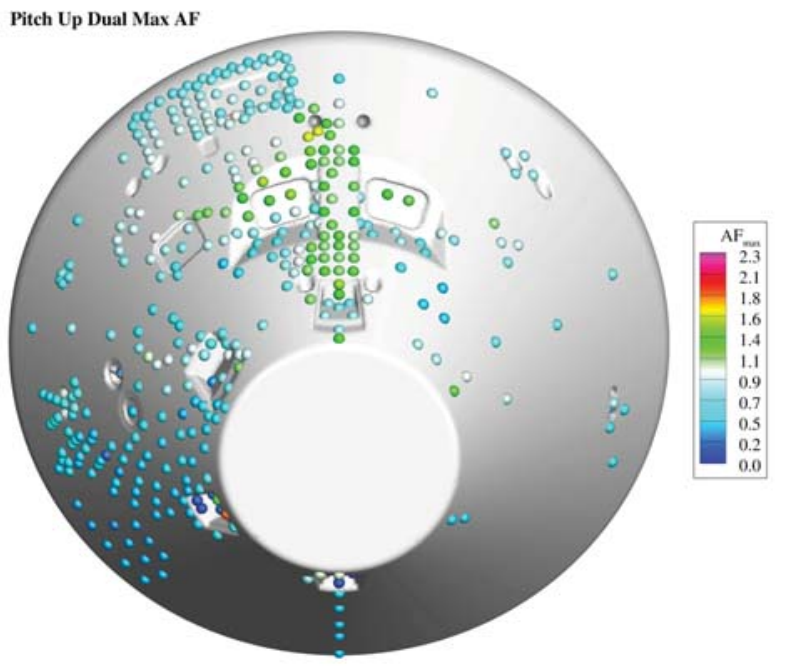

(b) Dual Pitch Up from 66B-CH

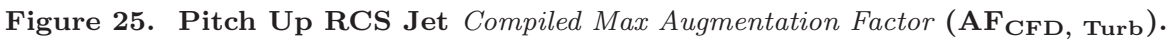

augmentation factor values within each region of the RCS jet maps.

Table 3. Summary of the augmentation factor values normalized by turbulent smooth OML CFD heating levels $\left(A F_{C F D}\right.$, turb) within each region of the RCS jet heating augmentation factor map areas

\begin{tabular}{|c|c|c|c|c|c|}
\hline RCS Jet Bank & Usage & Near Jet Region & Far-Field Region & Over-temp Region & Other \\
\hline \hline \multirow{3}{*}{ Roll } & Single & 4.7 & 3.6 & 1.5 & NA \\
\cline { 2 - 6 } & Dual & 8.2 & 4.5 & 3.0 & NA \\
\hline \multirow{3}{*}{ Pitch Down } & Single & 5.2 & 2.0 & NA & NA \\
\cline { 2 - 6 } & Dual & 5.2 & 2.0 & NA & NA \\
\hline \multirow{2}{*}{ Pitch Up } & Single & 2.3 & 1.6 & NA & NA \\
\cline { 2 - 6 } & Dual & 2.3 & 1.8 & NA & NA \\
\hline \multirow{2}{*}{ Yaw } & Single & 5.0 & 4.0 & NA & NA \\
\cline { 2 - 6 } & Dual & 5.0 & 4.0 & NA & NA \\
\hline \multirow{2}{*}{ Roll / Pitch Down } & Single & NA & NA & NA & 7.1 \\
\cline { 2 - 6 } & Single & NA & NA & NA & 7.1 \\
\hline
\end{tabular}

\section{Roll RCS Jets}

The single and dual roll augmentation factor maps are shown in Fig. 26. There is a higher rectangular shaped near jet augmentation factor region close to the nozzles, with an $\mathrm{AF}_{\mathrm{CFD}}$, turb of 4.7 and 8.2 for the single and dual firings respectively, which bounds the high near jet heating seen in the test data. The area is larger than the gauge locations that measured this near jet interaction to cover for the possibility of the interaction moving with changes in freestream and jet conditions throughout the trajectory as well as flight traceability uncertainty. The far-field area envelopes the remainder of the roll jet influence area, with the exception of a small sliver along the apex shoulder. The $\mathrm{AF}_{\mathrm{CFD}}$, turb in the far-field are lower than that of the near jet region at 3.6 and 4.5 for the single and dual jet firings respectively. The third area was added after initial thermal analysis predicted temperatures that exceeded the maximum allowable material 
temperature limit. As discussed in Section V.C, the general approach to defining regions within the map areas focused on a near jet region and second region that covered the remaining area, and that was the initial approach taken here. When that relatively conservative approach to defining the regions suggested a material change might be required, the available data in this region were re-evaluated and revealed the far-field region augmentation level to be overly conservative. A more appropriate $\mathrm{AF}_{\mathrm{CFD}}$, turb of 1.5 and 3.0 for the single and dual roll RCS jet firings respectively were developed for this region based on the data . The region was referred to as the "over-temp" region.

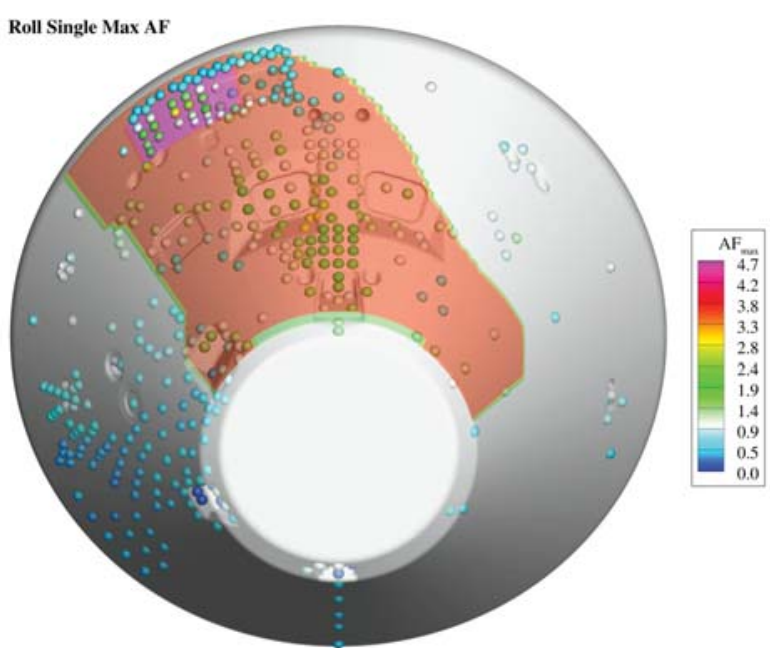

(a) Single Roll Right from 66B-CH / 123-CH Part A

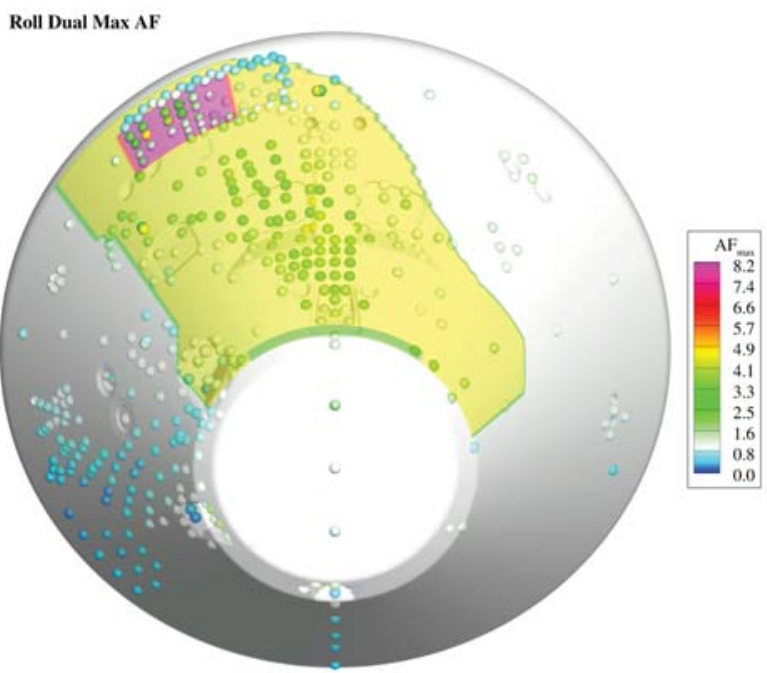

(c) Dual Roll Right from 66B-CH / 123-CH Part A

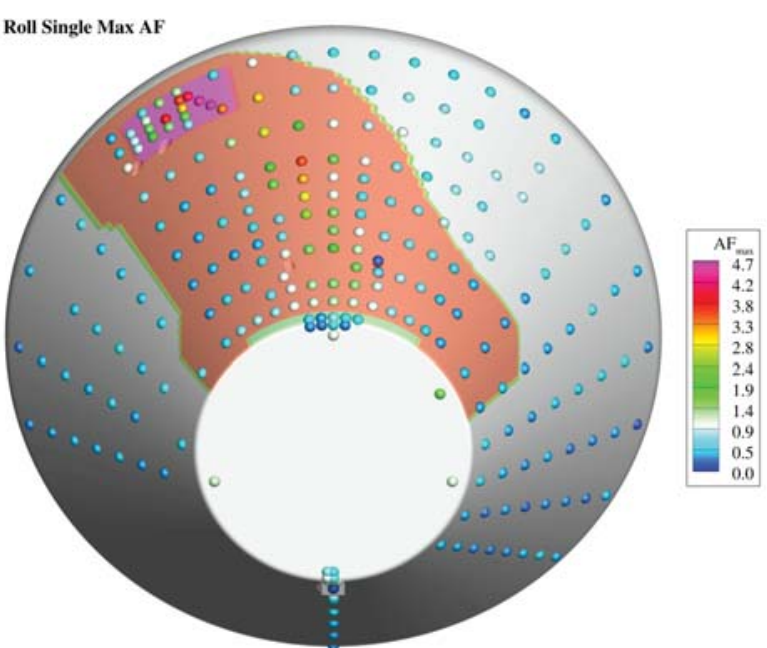

(b) Single Roll Right from 123-CH Part B

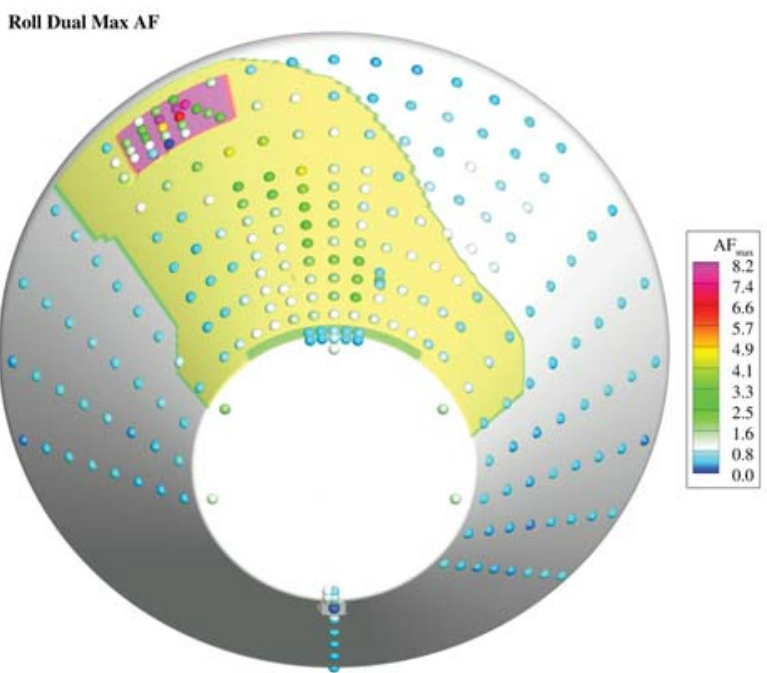

(d) Dual Roll Right from 123-CH Part B

Figure 26. Roll Right Maximum Compiled Results and Database Maps.

\section{Pitch Down RCS Jets}

The single and dual pitch down augmentation factor maps are shown in Fig. 27. The location of the high augmentation factors varied appreciably between the 66B-CH / 123-CH part A test article data and the 123-CH part B test article data. The differences could be associated with the change in the aft-body cone angles between the two test articles as well as the influence of the large window cavities. Given the variation of the peak augmentation factors, the high magnitude augmentation factor region was made to be a fairly 
large region with respect to the overall influence area. The map drops to a lower factor outside this region to reflect the smaller, but not negligible, augmentation factors elsewhere. Table 3 lists the augmentation factor magnitudes associated with each region. There is no difference in the magnitudes between the single and dual firing cases as the maximum augmentation factor seen in each was very similar.

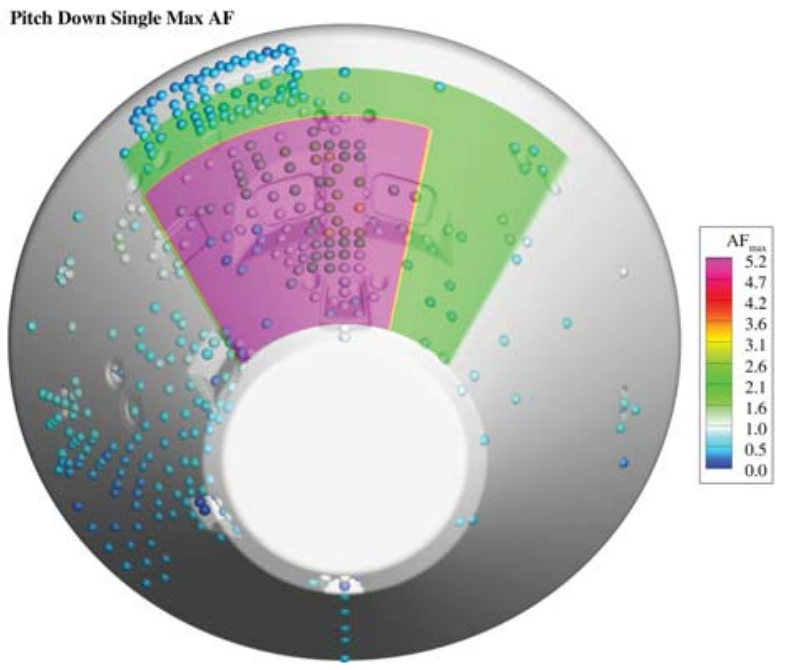

(a) Single Pitch Down from 66B-CH / 123-CH Part A

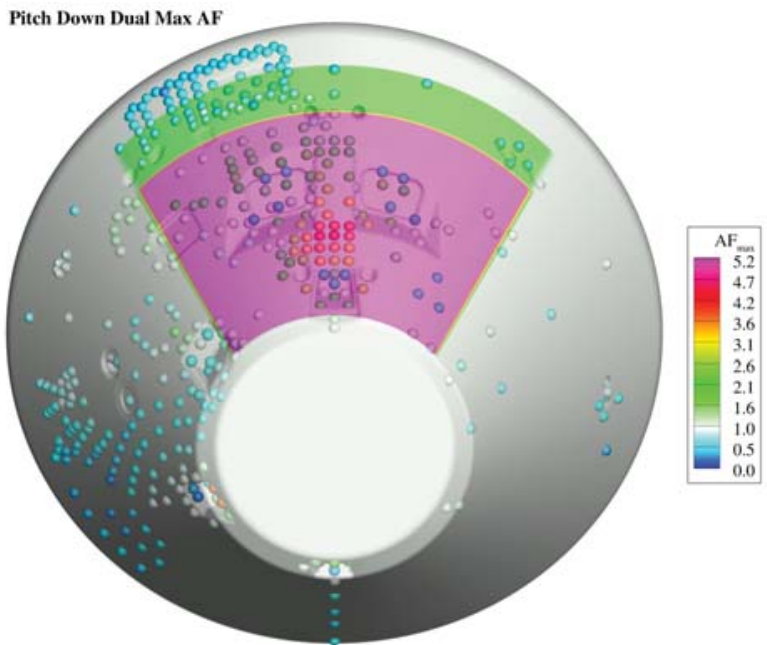

(c) Dual Pitch Down from 66B-CH / 123-CH Part A

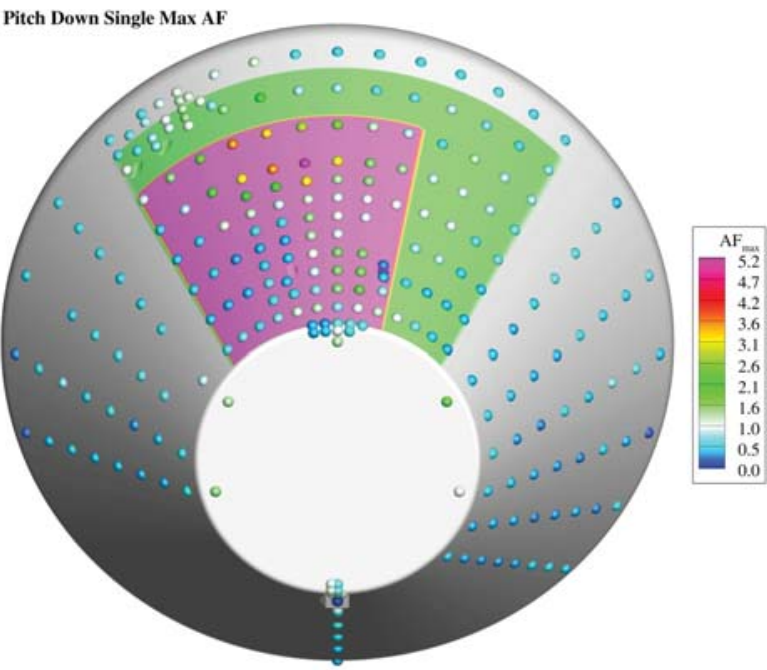

(b) Single Pitch Down from 123-CH Part B

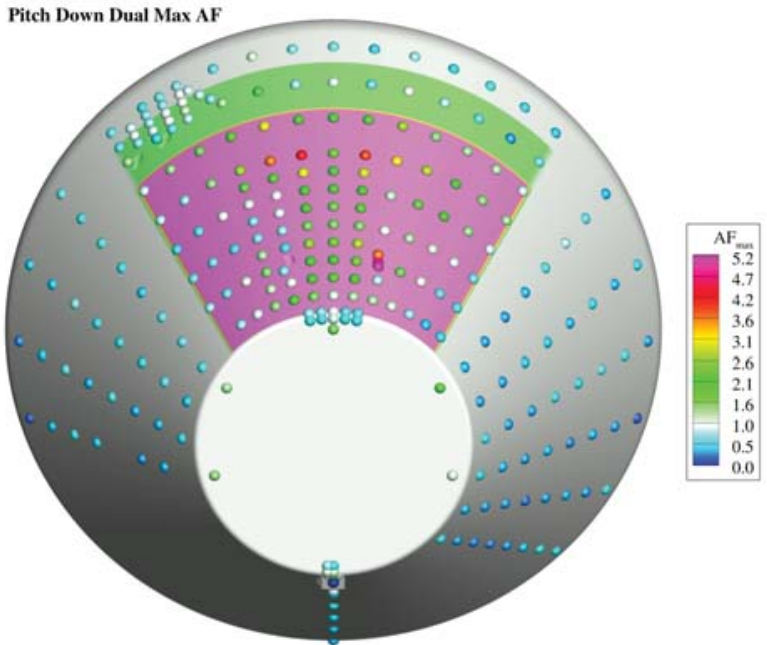

(d) Dual Pitch Down from 123-CH Part B

Figure 27. Pitch Down Maximum Compiled Results and Database Maps.

\section{3. $\quad$ Pitch Up RCS Jets}

The pitch up augmentation factor maps are shown in Fig. 28. These maps are very similar to the pitch down augmentation factor maps in that the single and dual magnitudes are very close, and the near jet augmentation factor is reduced on the opposite side of the body when only a single jet is firing. The near jet region is very small as the pitch up jet interaction is not very significant, and the higher factors are limited to the region very close to the nozzles. The associated $\mathrm{AF}_{\mathrm{CFD}}$, turb values are called out in Table 3 . 
Pitch Up Single Max AF

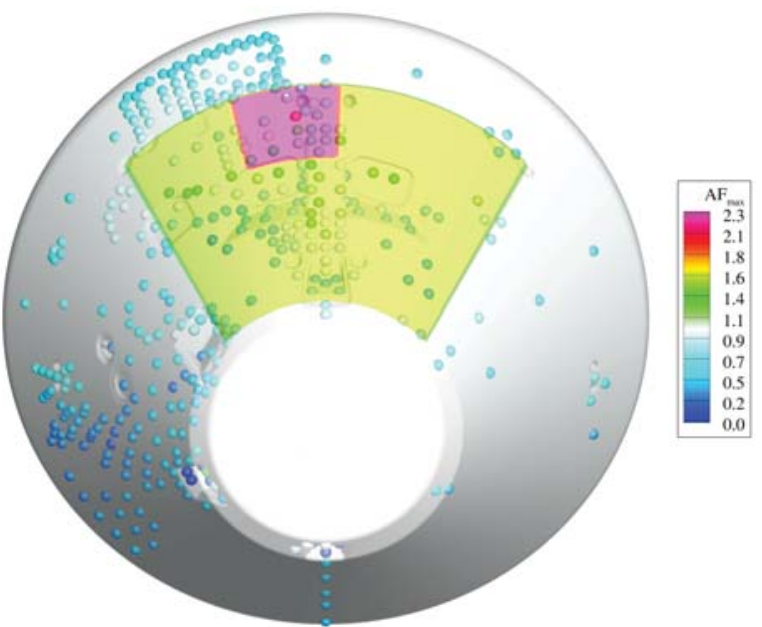

(a) Single Pitch Up from 66B-CH

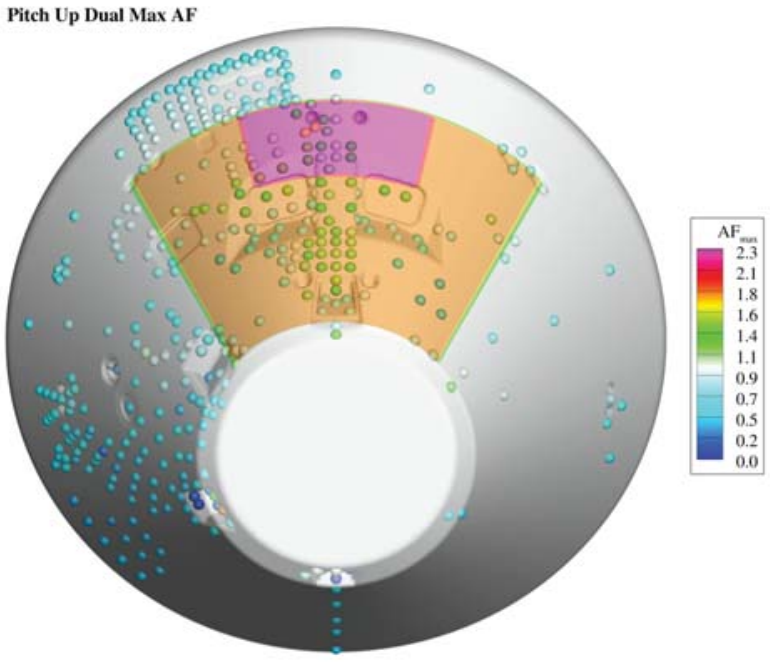

(b) Dual Pitch Up from 66B-CH

Figure 28. Pitch Up Maximum Compiled Results and Database Maps.

\section{Yaw RCS Jets}

In a deviation from the method outlined and used for all of the other jet banks, the yaw jet augmentation factor maps included some use of engineering judgment. The team acknowledged that the wind tunnel conditions tested did not cover all the expected scenarios in flight. Specifically, during the testing the yaw RCS jets were in the separated flow region, while in flight at certain angles of attack and certain flight regimes they were expected to be in the attached flow region. Given that this was an un-tested condition the map areas and magnitudes were increased beyond those that the Maximum Compiled Augmentation Factor approach coupled with the $\mathrm{AF}_{\mathrm{CFD}}$, turb formulation described in Eqn. (7) indicated. These maps are shown overlaid with the maximum compiled yaw jet data in Fig. 29 and the associated $\mathrm{AF}_{\mathrm{CFD}}$, turb values are listed in Table 3.

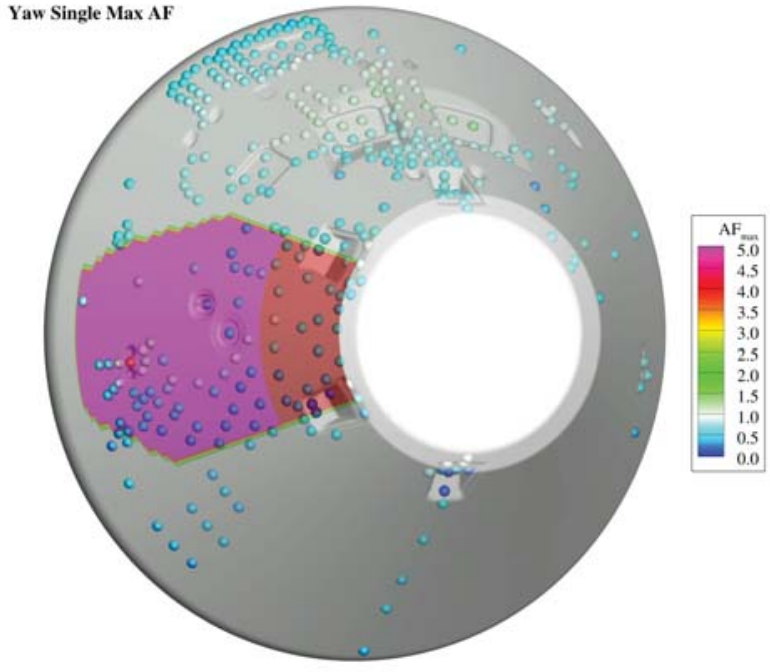

(a) Single Yaw from 66B-CH

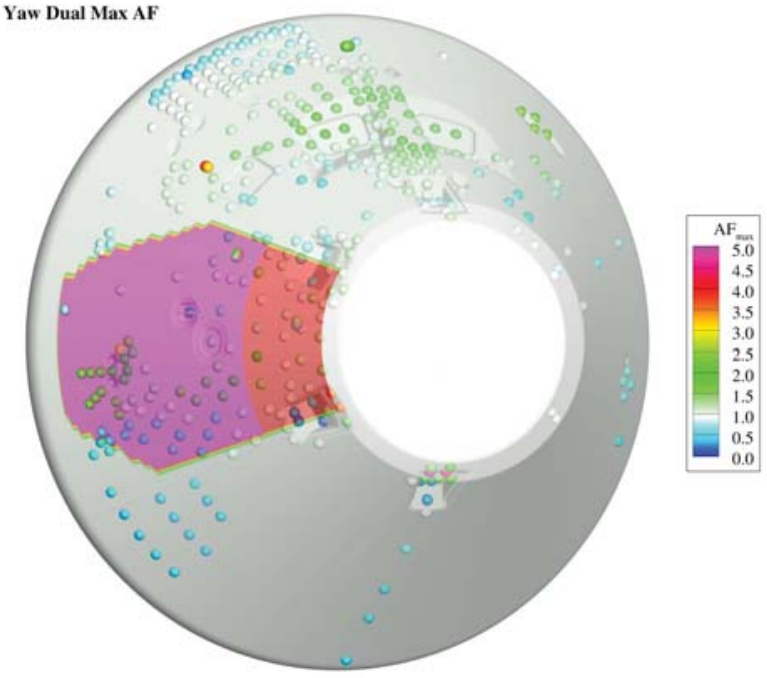

(b) Dual Yaw from 66B-CH

Figure 29. Yaw Maximum Compiled Results and Database Maps. 


\section{Combinations of RCS Jets Fired Simultaneously}

When RCS jets are fired in combination, the associate individual RCS jet maps are combined by taking the maximum at all locations. The data corroborated this except for when the roll RCS jets fire in combination with the pitch down RCS jets. The data showed that a small sub region within the combined roll and pitch down influence areas exceeded the heating augmentation level measured when either individual jet was fired alone, as described in Section IV.D. To accommodate this, an additional map was developed to bound that region, shown in Fig. 30 overlaid with the dual roll / pitch down data. The $\mathrm{AF}_{\mathrm{CFD}}$, turb in this region of 7.1 was called out in Table 3 as other.

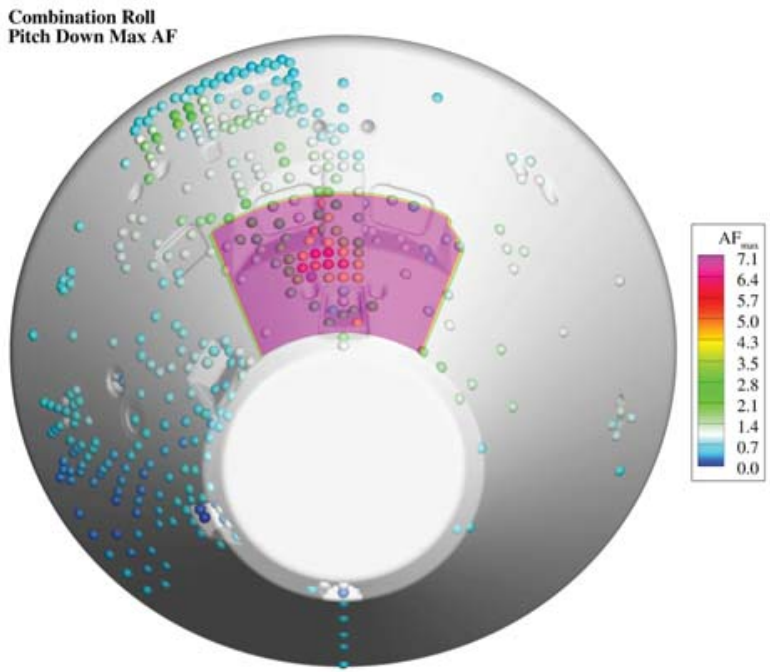

(a) Combination Roll Right and Pitch Down from 66B-CH / 123-CH Part A

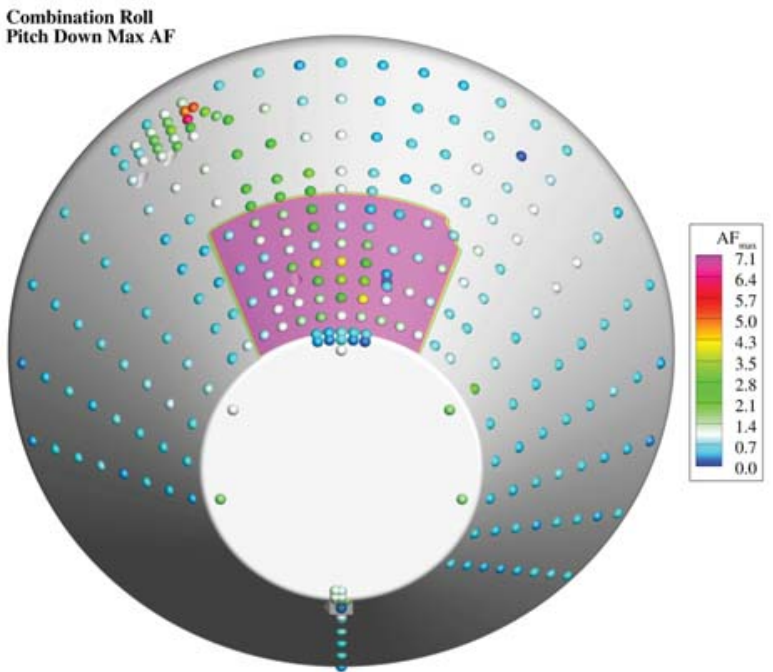

(b) Combination Roll Right and Pitch Down from 123-CH Part B

Figure 30. Combination Maximum Compiled Results and Database Maps.

\section{Conclusions}

Data from a series of wind tunnel tests, conducted to support the development of the RCS jet augmentation factor models for the Orion MPCV, have been evaluated in the present work. As part of those tests a number of potential RCS jet similarity parameters have been examined including the jet-to-freestream momentum ratio and enthalpy ratio. The qualitative trends observed in the test data have been identified. These include trends in the RCS jet heating augmentation level with the jet-to-freestream momentum ratio when the roll and pitch down RCS jets where fired. Several lessons learned were also identified, including increased heating augmentation when certain jet combinations were fired as compared to the associated jets firing independently and changes to certain cavity augmentation environments when the roll jets were fired. The data also revealed that the RCS jet environments can be very localized and discrete instrumentation can fail to capture peaks in these environments.

The data revealed heating augmentation factors as high as 13.0, 7.6, 2.8, and 5.8 for the roll, pitch down, pitch up, and yaw jets firing respectively. In the case of the roll and yaw RCS jets, these levels are similar to those measured during the design of the Apollo vehicle.

Finally, a series of augmentation factor maps have been developed to be used in combination with the SOML predicted heating to provide RCS environments for the design of the Orion MPCV. The version of the RCS jet augmentation factor maps presented here are those used for the design of the first test flight (EFT-1) of the Orion MPCV. The maps were developed without taking advantage of potential relief from many of the observed trends in the wind tunnel test data due to the unknown impact of the chemically reacting freestream interacting with the reacting RCS jet plume gases, other ground to flight traceability effects, and general limitations associated with wind tunnel testing, like the impact of the sting.

There are still a number of open questions, in particular, ground-to-flight traceability. It is hoped that 
in the future the EFT-1 flight data will help resolve the traceability question as well as help understand the potential jet-to-freestream similarity parameters and specifically the trends observed in the wind tunnel test data or help identify other similarity parameters.

\section{Acknowledgments}

The authors thank all of the test Principal Investigators (PI), Randy Lillard and Ben Kirk of NASA Johnson Space Center and Greg Buck of the NASA Langley Research Center for their part in designing and executing the experimental campaigns that made this work possible, as well as Tim Wadhams of the CUBRC test facility for his support and insight in designing the tests conducted at CUBRC. Thanks also to Artem Dyakonov, now working for Blue Origin, LLC., for helping to design the 66B-CH test and in particular for leading the RCS aspects of the design. Special thanks to Joseph Bomba of Lockheed Martin Space Systems Company and Adam Amar of NASA Johnson Space Center for numerous conversations and endless support with the development of the augmentation models themselves. Additionally, the authors thank Amy Cassady of NASA Johnson Space Center for helping to find the funding necessary to execute all of the tests and for leading the development of the entire aerothermal database. Finally, the authors would like to thank the entire aerothermal aerosciences team for supporting this effort over the years. This work was completed, in part, under the NASA Ames Research Center contract number NNA10DE12C to ERC Incorporated.

\section{References}

\footnotetext{
${ }^{1}$ Greathouse, James S., Kirk, Benjamin S., Lillard, Randolph P., Truong, Tuan H., Robinson, Phil, and Cerimele, Chris J., "Crew Exploration Vehicle (CEV) Crew Module shape selection analysis and CEV Aerosciences Project Overview," $45^{t h}$ AIAA Aerospace Sciences Meeting and Exhibit, 8 - 11 January 2007, Reno, NV

${ }^{2}$ Kinney, David J., "Development of the ORION Crew Exploration Vehicle's Aerothermal Database Using A Combination of High Fidelity CFD and Engineering Level Methods," 47 th AIAA Aerospace Sciences Meeting and Exhibit, 5 - 8 January 2009, Orlando, FL

${ }^{3}$ Jones, Robert A. and Hunt, James L., "Effects of Cavities, Protuberances, and Reaction-Control Jets on Heat Transfer to the Apollo Command Module," NASA TM X-1063, March 1965

${ }^{4}$ Dyakonov, Artem A., Buck, Gregory M., and DeCaro, Anthony D., "Analysis of Aeroheating Augmentation due to Reaction Control System Jets on Orion Crew Exploration Vehicle," 41 ${ }^{\text {st }}$ AIAA Thermophysics Conference, 22 - 25 June 2009 , San Antonio, TX

${ }^{5}$ Wright, Michael J., Prabhu, Dinesh K., and Martinez, Edward R., "Analysis of Apollo Command Module Afterbody Heating Part I: AS-202," Journal of Thermophysics and Heat Transfer

${ }^{6}$ Tang, Chun, "Simulations of Afterbody Heating Rates on the Apollo Command Module for AS-202 using Hyperbolic Grids," $9^{t h}$ AIAA/ASME Joint Thermophysics and Heat Transfer Conference, 5-8 June 2006, San Francisco, CA

${ }^{7}$ Barnhardt, Michael, Candler, Graham V., and MacLean, Matthew, "CFD Analysis of CUBRC Base Flow Experiments," $48^{\text {th }}$ AIAA Aerospace Sciences Meeting, 4 - 7 January 2010, Orlando, FL

${ }^{8}$ Salazar, Giovanni and Edwards, Jack R., "Numerical Simulation of CUBRC Wake Flow Experiments Using a Hybrid LES/RANS Approach," 51 $1^{\text {st }}$ AIAA Aerospace Sciences Meeting, 7 - 10, January 2013, Dallas, TX

${ }^{9}$ Cassel, L. A., "Applying Jet Interaction Technology," Journal of Spacecraft and Rockets, Vol 40, No. 4, July-Aug. 2003, pp. 523-537. doi:10.2514/2.3992

${ }^{10}$ Spaid, F. W., and Cassel, L. A., "Aerodynamic Interference Induced by Reaction Controls," AGARDograph, AGARDAG-173, NATO, Dec. 1973

${ }^{11}$ Ivey, Christopher B., Danehy, Paul M., Bathel, Brett F., Dyakonov, Artem A., Inman, Jennifer A., and Jones, Stephen B., "Comparisons of PLIF and CFD Results for the Orion CEV RCS Jets," $49^{\text {th }}$ AIAA Aerospace Sciences Meeting, 4 - 7 , January 2011, Orlando, FL

${ }^{12}$ Combs, C. S., Clemens, N. T., Danehy, P. M., Bathel, B., Parker, R., Wadhams, T., Holden, M., and Kirk, B., "NO PLIF Visualizations of the Orion Capsule in LENS-1," 51 ${ }^{\text {st }}$ AIAA Aerospace Sciences Meeting, 7 - 10, January 2013, Dallas, TX

${ }^{13}$ Ben-Yakar, A., Mungal, G. M., and Hanson, R. K., "Time Evolution and Mixing Characteristics of Hydrogen and Ethylene Transverse Jets in Supersonic Crossflows," Physics of Fluids, Vol 18, No. 2, February 2006, Paper 026101

${ }^{14}$ M. R. Gruber, A. S. Nejad, T. H. Chen, and J. C. Dutton, "Mixing and penetration studies of sonic jets in a Mach 2 freestream, J. Propul. Power 0748-4658 11, 315 (1995)

${ }^{15}$ Anderson, John D., Modern Compressible Flow with Historical Perspective, Third Edition, McGraw Hill, New York, NY, 2003

${ }^{16}$ Buck, Gregory M., Watkins, A. Neal, Danehy, Paul M., Inman, Jennifer A., Alderfer, David W., Dyakonov, Artem A., "Experimental Measurement of RCS Jet Interaction Effects on a Capsule Entry Vehicle," $46^{\text {th }}$ AIAA Aerospace Sciences Meeting and Exhibit, 7 - 10 January 2008, Reno, NV.

${ }^{17}$ Buck, Gregory M., "Aerothermodynamic Testing of RCS Jet Interactions for the Orion 606D Crew Module in the NASA Langley 31-Inch Mach 10 Air Tunnel: Test 63-CH," NASA Johnson Space Center, EG-CAP-08-173 (Restricted), October 2008
} 
${ }^{18}$ Wadhams, T. P., MacLean, M. G., Holden, M. S., "Experimental Studies of Spherical Capsule Wake Flows with Reaction Control Jet Interaction," 52 ${ }^{\text {nd }}$ AIAA Aerospace Sciences Meeting, 13 - 17 January 2014, National Harbor, MD

${ }^{19}$ Buck, Gregory M., "Aerothermodynamic Testing of RCS Jet Interactions for the Orion 607A Crew Module in the NASA Langley 31-Inch Mach 10 Air Tunnel: Test 95-CH," NASA Johnson Space Center, EG-CAP-11-95 (Restricted), November 2011 